\title{
PUZZLES OF QUASI-FINITE TYPE, ZETA FUNCTIONS AND SYMBOLIC DYNAMICS FOR MULTI-DIMENSIONAL MAPS
}

by

Jérôme Buzzi

\begin{abstract}
Contents
1. Introduction $\ldots \ldots \ldots \ldots \ldots \ldots \ldots \ldots \ldots \ldots \ldots \ldots \ldots \ldots \ldots \ldots \ldots, 1$

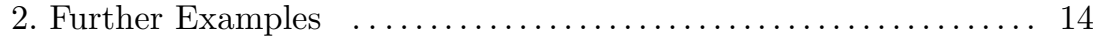

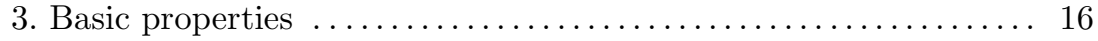

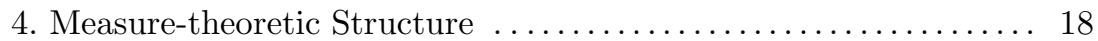

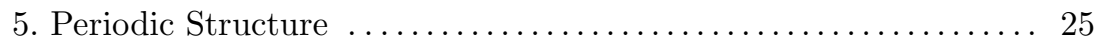

6. Semi-local zeta functions of SPR Markov shifts $\ldots \ldots \ldots \ldots \ldots .28$

7. Proof of the Consequences ............................ 30

8. Application to entropy-expanding maps $\ldots \ldots \ldots \ldots \ldots \ldots \ldots \ldots \ldots$

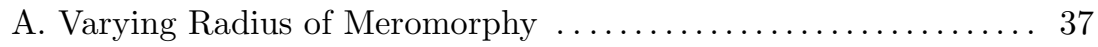

B. Good Partitions for Almost All Couplings . .............. 39

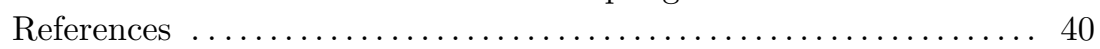

\section{Introduction}

In what sense(s) can a dynamical system be "complex" and what is the interplay between this complexity and the more classical dynamical properties? A very large body of works has been devoted to this basic question, especially to prove various forms of complexity from dynamical assumptions. We are interested in reversing this direction:

What are the dynamical consequences of complexity?

Can complexity characterize a dynamical system? 
This type of question has been studied mainly in low-complexity settings (see, e.g., 1 and the references therein). We have shown, first in a smooth setting, that a high-complexity assumption (which we called entropy-expansion) also has very thorough dynamical implications [9. A remarkable feature is that this condition, which involves only so-called dimensional entropies, is enough to analyze measures of maximum entropy and the related periodic points. We are even able to classify such systems with respect to all their ergodic and invariant measures of high entropy. Thus complexity can be analyzed using only (simple) complexity assumptions.

The proofs in 9 mix both combinatorial/entropic arguments and geometric ones involving Lyapunov exponents, the smoothness and the ensuing approximations by polynomials, raising the question of separating completely both issues. In [11, we achieved this separation for, e.g., subshifts of finite type, piecewise monotonic interval maps with nonzero entropy, and multidimensional $\beta$-transformations giving a common proof to their common "complexity" properties. However the estimate required by 11] seems tractable only when cylinders are connected, preventing until now the application of these constructions to multi-dimensional, non-linear, entropy-expanding maps.

The present paper overcomes this obstacle (see the remark after Prop. 8.1) by introducing a suitable type of symbolic dynamics which we call puzzles of quasi-finite type -these are puzzles in the sense of Yoccoz' construction in complex dynamics. In comparison with our work in $\mathbf{9}$ we have to make an additional, probably generic, assumption, but we also obtain more detailed information about the periodic points.

The puzzle of quasi-finite type are the generalization of the subshifts of quasi-finite type 11 needed for multi-dimensional, non-linear maps (see the end of section 1.3). We generalize to these puzzles all the results obtained for subshifts:

- existence of a finite number of ergodic probability measures maximizing the entropy;

- meromorphic extension of (suitably defined) Artin-Mazur zeta functions counting the periodic points;

- equidistribution of the periodic points;

- classification with respect to measure of large entropy.

The meromorphic extension is deduced from a new, similar result about Markov shifts (Theorem 4) relating the radius of meromorphy of some zeta functions of Markov shifts to their entropy at infinity (see Definition 1.10). This is of independent interest.

1.1. Definitions. - We recall the notion of a puzzle due to Yoccoz [27] (closely related are the tableaux of Branner and Hubbard).

Definition 1.1. - A puzzle is $(V, i, f)$ (or just $V$ ), a set of pieces $V=\sqcup_{n \geq 0} V_{n}$ (a disjoint union of finite sets) and two maps $i, f: V \backslash V_{0} \rightarrow V$ satisfying:

- $V_{0}$ has a single element;

$-i \circ f=f \circ i$

$-i\left(V_{n+1}\right) \subset V_{n}$;

$-f\left(V_{n+1}\right) \subset V_{n}$.

The order of a piece $v$ is $|v|$, the unique integer $n$ such that $v \in V_{n}$. 
The above combinatorial data defines a topological dynamics as follows:

Definition 1.2. - A puzzle $(V, i, f)$ defines the dynamics $F_{V}: X_{V} \rightarrow X_{V}$ :

$$
\begin{gathered}
X_{V}=\left\{v \in V_{0} \times V_{1} \times \cdots: \forall n \geq 0 i\left(v_{n+1}\right)=v_{n}\right\} \\
F_{V}:\left(v_{n}\right)_{n \geq 0} \longmapsto\left(f\left(v_{n+1}\right)\right)_{n \geq 0} .
\end{gathered}
$$

1.2. Some examples. - For $v \in V$, we denote by $|v|$ the unique integer $n$ such that $v \in V_{n}$. It is the order of $v$.

Subshifts are Puzzles. - Let $\sigma: \Sigma_{+} \rightarrow \Sigma_{+}$be an arbitrary one-sided subshift (i.e., a closed shift-invariant subset of $\mathcal{A}^{\mathbb{N}}$ for some finite set $\mathcal{A}$, the shift being $\sigma:\left(A_{n}\right)_{n \geq 0} \mapsto$ $\left.\left(A_{n+1}\right)_{n \geq 0}\right)$. We are going to define a puzzle $V$ such that the dynamics $F_{V}$ associated to $V$ is topologically conjugate to this subshift $\Sigma_{+}$.

For each $n \geq 0$, let $V_{n}$ be the set of words of length $n$ that appear in $\Sigma_{+}$, i.e., sequences $x_{0} \ldots x_{n-1}$ such that $y_{i+k}=x_{i}$ for $i=0, \ldots, n-1$ for some $y \in X$ (by convention, $V_{0}=\{\varnothing\}$ where $\varnothing$ is the empty word). Define the two maps $i$ and $f$ by:

$$
\begin{array}{cl}
i\left(A_{1} \ldots A_{n}\right)=A_{1} \ldots A_{n-1} & \text { rightmost delete } \\
f\left(A_{1} \ldots A_{n}\right)=A_{2} \ldots A_{n} & \text { leftmost delete. }
\end{array}
$$

The conjugacy $h: X_{V} \rightarrow \Sigma_{+}$is given by $h\left(\left(A_{1} \ldots A_{n}\right)_{n \geq 0}\right)=\left(A_{n+1}\right)_{n \geq 0}$.

From dynamics to puzzles. - Let $T: M \rightarrow M$ be a self-map. Let $\mathcal{P}_{0}=\{M\}, \mathcal{P}_{1}, \mathcal{P}_{2} \ldots$ be a sequence of finite partitions of $M$ satisfying

$$
\mathcal{P}_{n} \preceq \mathcal{P}_{n+1} \text { and } T^{-1} \mathcal{P}_{n} \preceq \mathcal{P}_{n+1}
$$

(where $\mathcal{P} \preceq \mathcal{Q}$ means that $\mathcal{P}$ is less fine than $\mathcal{Q}$ : each element of $\mathcal{P}$ is a union of elements of $\mathcal{Q}$; also $\left.T^{-1} P:=\left\{T^{-1} A: A \in P\right\}\right)$.

This data defines a puzzle as follows. Let $V$ be the disjoint union of $V_{n}=\mathcal{P}_{n}$, $n \geq 0$. Let $i(v)=w$ if $w$ is the element of $\mathcal{P}_{n}$ containing $v \in \mathcal{P}_{n+1}$. Let $f(v)=w$ if $w$ is the element of $\mathcal{P}_{n}$ containing $T(v)$ for $v \in \mathcal{P}_{n+1}$. The above assumptions ensure that this is a well-defined puzzle.

Let us give several examples of this construction.

- Let $\mathcal{P}$ be some finite partition and let $\mathcal{P}_{n}=\mathcal{P} \vee T^{-1} \mathcal{P} \vee \cdots \vee T^{-n+1} \mathcal{P}$. The corresponding puzzle is topologically conjugate to the usual symbolic dynamics, i.e., the left shift, $\sigma:\left(A_{n}\right)_{n \geq 0} \mapsto\left(A_{n+1}\right)_{n \geq 0}$ acting on:

$$
\overline{\left\{A \in \mathcal{P}^{\mathbb{N}}: \exists x \in M \forall n \geq 0 T^{n} x \in A_{n}\right\}} \subset \mathcal{P}^{\mathbb{N}} .
$$

- Let $\mathcal{P}$ be some finite partition and let $\mathcal{P}_{n}$ be the set of connected components of the elements of $\mathcal{P} \vee T^{-1} \mathcal{P} \vee \cdots \vee T^{-n+1} \mathcal{P}$. This is the form used in complex dynamics 27.

- The following is easy but important:

Fact 1.3. - Any continuous map $T$ on a Cantor set $K$ can be realized, up to topological conjugacy, as a puzzle, that is, there exist a puzzle $V$ and a homeomorphism $\phi: X_{V} \rightarrow K$ with $\phi \circ F_{V}=T \circ \phi$. 
ProOF: Let $\mathcal{Q}_{n}, n \geq 1$, be a sequence of partitions of $K$ into closed-open sets with diameters going to zero. Let $\mathcal{P}_{n+1}=\mathcal{Q}_{n+1} \vee \mathcal{P}_{n} \vee T^{-1} P_{n}$. It is then easy to see that the dynamics of the puzzle thus defined is conjugate to that of $T$.

Thus, the dynamics of puzzles are even more diverse than that of subshifts. For instance, they can have infinite entropy or be without measures of maximum entropy. To get a tractable class we shall assume some form of "simplicity".

1.3. Notions of simplicity. - We restrict ourselves to puzzles that are defined by "few constraints" (and this will include subshifts of finite type as the special case of finitely many "constraints"). The following choice of a notion of "simplicity" turns out to allow a detailed analysis and more precisely enforces a close similarity to the classical properties of subshifts of finite type.

A notion of constraint: irreducibility. — Let the $i$-tree below $v \in V$ be the directed graph $\mathcal{T}_{i}(v)$ whose vertices are the $w \in V$ such that

$$
i^{n}(w):=\underbrace{i \circ \cdots \circ i}_{n \text { factors }}(w)=v
$$

for some $n \geq 0$ and whose edges are $u \rightarrow u^{\prime}$ iff $u^{\prime}=i(u)$.

Definition 1.4. - A piece $v \in V \backslash V_{0}$ is f-reducible if the two following conditions hold:

(R1) $f: \mathcal{T}_{i}(v) \rightarrow \mathcal{T}_{i}(f(v))$ is a graph isomorphism;

(R2) there is no $w \neq v$ such that $i(w)=i(v), f(w)=f(v)$ and (R1) holds also for $w$.

Otherwise, $v$ is said to be $f$-irreducible.

Notations. $v \succeq_{f}^{1} w$ means that $v$ is $f$-reducible and $w=f(v)$. For $k>1, v \succeq_{f}^{k} w$ is defined inductively as $v \succeq_{f}^{1} f(v)$ and $f(v) \succeq_{f}^{k-1} w$ (by convention $v \succeq_{f}^{0} v$ for any $v \in V)$. Finally $u \succeq_{f} w$ means that $u \succeq_{f}^{k} w$ for some $k \geq 1$.

Remark. Property (R1) was introduced by Yoccoz under the name of "regularity" . It is equivalent to the following dynamical property (here $[v]_{V}:=\left\{x \in X_{V}: x_{|v|}=v\right\}$ ):

$$
F_{V}:[v]_{V} \rightarrow[f(v)]_{V} \text { is a bijection. }
$$

In the setting of complex dynamics, failure of (R1) is equivalent to containing critical points and is called criticality -see [5].

Condition (R2) seems new. It is often a consequence of (R1) -this is the case, if, for instance, the restrictions $F_{V} \mid[v]_{V}, v \in V$, are one-to-one.

Remark. In the case where the puzzle is given by a subshift as in section 1.2, condition (R2) of Definition 1.4 is automatically satisfied (indeed, $f(v)=f(w)$ and $i(v)=i(w)$ imply $v=w$ ) and condition (R1) is equivalent to the notion of a minimum left constraint introduced for subshifts $\Sigma$ in [11]: it is a finite word $w_{-n} \ldots w_{0}$ such that:

$$
\begin{aligned}
\left\{x_{0} x_{1} \cdots: x \in \Sigma \text { s.t. } x_{-n} \ldots x_{0}=w_{-n} \ldots w_{0}\right\} & \\
& \subsetneq\left\{x_{0} x_{1} \cdots: x \in \Sigma \text { s.t. } x_{-n+1} \ldots x_{0}=w_{-n+1} \ldots w_{0}\right\} .
\end{aligned}
$$


One can understand the $f$-irreducible pieces as describing the constraints in $X_{V}$. The $f$-reducibility of some $v$ implies that the possible $i$-extensions of $v$ are the same as those of $f(v)$. This is a sort of "local Markov property". For instance, if all pieces are reducible then $X_{V}=V_{1}^{\mathbb{N}}$. A slightly less extreme example of this phenomenon is the following Lemma proved in section 2.1 .

Lemma 1.5. - If a puzzle $V$ has only finitely many $f$-irreducible vertices, then $X_{V}$ is (topologically conjugate to) a subshift of finite type, i.e., a subshift of $\{1,2, \ldots, d\}^{\mathbb{N}}$ for some $d \geq 1$, obtained by excluding a finite number of finite sequences [39].

More generally, one can expect puzzles with few $f$-irreducible vertices to be "simple". The definition below formalizes this idea.

Entropy on the puzzle. — The puzzle $V$ will be equipped with the following combinatorial distance: for $v \neq w$,

$$
d_{V}(v, w)=2^{-n} \quad \text { if } n=\max \left\{0 \leq k \leq \min (|v|,|w|): i^{|v|-k}(v)=i^{|w|-k}(w)\right\} .
$$

Note that $d_{V}(i(v), i(w)) \leq 2 d_{V}(v, w)$ and $d_{V}(f(v), f(w)) \leq 2 d_{V}(v, w)$.

The corresponding metric on $X_{V}$ is

$$
d_{V}(x, y):=\sup _{n \geq 0} d_{V}\left(x_{n}, y_{n}\right)=2^{-n} \text { such that } n=\min \left\{k \geq 0: x_{k} \neq y_{k}\right\} \text { or } \infty \text {. }
$$

Together with $f$, this induces a notion of Bowen balls in $V$ : for $v \in V, \epsilon>0$, $n \in \mathbb{N}$, the $(\epsilon, n)$-ball around $v$ is

$$
B(v, \epsilon, n):=\left\{w \in V: \forall 0 \leq k<\min (n,|v|,|w|) d_{V}\left(f^{k} w, f^{k} v\right)<\epsilon\right\} .
$$

The covering number $r(\epsilon, n, S)$ is the minimum number of $(\epsilon, n)$-balls needed to cover $S \subset V$. We define the topological entropy of a sequence $\mathcal{S}$ of subsets $S_{n} \subset V_{n}, n \geq 1$, as:

$$
h_{\text {top }}(\mathcal{S})=\lim _{\epsilon \rightarrow 0} \limsup _{n \rightarrow \infty} \frac{1}{n} \log r\left(\epsilon, n, S_{n}\right) .
$$

We recall first Bowen-Dinaburg formula for the topological entropy. The $(\epsilon, n)$ Bowen ball at $x$ w.r.t. $F_{V}$ (and a distance $d$ on $X_{V}$ ), is $B(x, \epsilon, n):=\left\{y \in X_{V}: \forall k<n\right.$ $\left.d\left(F_{V}^{k} x, F_{V}^{k} y\right)<\epsilon\right\}$. The topological entropy 39 is

$$
h_{\text {top }}\left(F_{V}\right):=\lim _{\epsilon \rightarrow 0} h_{\text {top }}\left(F_{V}, \epsilon\right) \text { with } h_{\text {top }}\left(F_{V}, \epsilon\right)=\limsup _{n \rightarrow \infty} \frac{1}{n} \log r\left(\epsilon, n, X_{V}\right)
$$

where $r(\epsilon, n, S)$ is the minimum number of $(\epsilon, n)$-balls necessary to cover $S$. We sometimes write $h_{\mathrm{top}}(V)$ instead of $h_{\mathrm{top}}\left(F_{V}\right)$.

Let $\mu$ be a probability measure $\mu$ on $X_{V}$ which is ergodic and invariant under $F_{V}$. The Kolmogorov-Sinai entropy of $\left(F_{V}, \mu\right)$ can be defined as follows, according to Katok (we again refer to 39 for background):

$$
h\left(F_{V}, \mu\right):=\lim _{\epsilon \rightarrow 0} h\left(F_{V}, \mu, \epsilon\right) \text { with } h\left(F_{V}, \mu, \epsilon\right)=\limsup _{n \rightarrow \infty} \frac{1}{n} \log r(\epsilon, n, \mu)
$$

where $r(\epsilon, n, \mu)$ is the minimum number of $(\epsilon, n)$-balls whose union has $\mu$-measure at least $1 / 2$ (it can be proved that $1 / 2$ can be replaced with any number in $(0,1)$ without affecting $\left.h\left(F_{V}, \mu\right)\right)$. 
JÉRÔME BUZzI

Constraint entropy. - We now state our main condition on the complexity of puzzles

Definition 1.6. - The constraint entropy of a puzzle $V=(V, i, f)$ is:

$$
h_{\mathcal{C}}(V):=h_{\text {top }}\left(\left(C_{n}\right)_{n \geq 1}\right)
$$

where $C_{n}$ is the set of irreducible pieces of order $n$.

The puzzle $V$ is of *-quasi-finite type (or, for short, *-QFT) if it satisfies:

$$
h_{\mathcal{C}}(V)<h_{\text {top }}(V) .
$$

This notion is essentially unrelated to that of subshifts of weakly quasi-finite type defined in [1].

W-Local entropy. - *-QFT puzzles can still present complexity at arbitrarily small scales. For example one can build $*$-QFT puzzles which are the union of sequences of subshifts of finite type with equal or increasing entropy so that they have either infinitely many or no ergodic invariant probability of maximum entropy - see section 2.2 .

Our second restriction prevents these phenomena.

Definition 1.7. - $V$ being a *-QFT puzzle, the W-local entropy of $V$ is the defect in uniformity of $h\left(F_{V}, \mu\right)=\lim _{\epsilon \rightarrow 0} h\left(F_{V}, \mu, \epsilon\right)$ over large entropy measures (that is, ergodic invariant probability measures with entropy close to the supremum):

$$
h_{\text {wloc }}(V):=\inf _{\epsilon>0} \sup _{\mu} h\left(F_{V}, \mu\right)-h\left(F_{V}, \mu, \epsilon\right)
$$

where $\mu$ ranges over the ergodic invariant probability measures on $X_{V}$ with entropy $>h_{\mathcal{C}}(V)$.

Remark.

Obviously, $h_{\text {wloc }}(V) \leq h_{\text {loc }}\left(F_{V}\right)$, the local entropy (introduced by Misiurewicz [30]) under the name topological conditional entropy) which bounds this defect in uniformity over all measures. In particular, $h_{\mathrm{wloc}}(V)=0$ if $F_{V}$ is expansive, e.g., a subshift.

Definition 1.8. - A QFT puzzle (or just QFT) is a puzzle $(V, i, f)$ which satisfies:

$$
h_{\mathcal{C}}(V)+h_{\text {wloc }}(V)<h_{\text {top }}(V) .
$$

The notions of QFT and *-QFT puzzles can be readily generalized in the following fashion (adding new examples - as this already happens for subshifts, see [11]). Observe that if $(V, i, f)$ is a puzzle, then so is: $\left(V^{*}, i^{*}, f^{*}\right)$ with $V^{*}=V, i^{*}=f$ and $f^{*}=i .\left(V^{*}, i^{*}, f^{*}\right)$ is called the dual puzzle. The dynamics of a puzzle and its dual are closely related. Hence one could formally extends our theorems by assuming that their assumptions hold either for the puzzle or for its dual (see Sec. 3.2). 
Determinacy. - The analysis of periodic points uses a further assumption. We state it in terms of the projections, for $N=1,2, \ldots$,

$$
i_{N}: V \rightarrow \bigcup_{k \leq N} V_{k}, v \mapsto i^{(|v|-N)^{+}}(v) .
$$

$i_{N}$ extends to a map $i_{N}: X_{V} \rightarrow\left(\bigcup_{k \leq N} V_{k}\right)^{\mathbb{N}}$ in a natural way:

$$
i_{N}(x)=y \Longleftrightarrow \forall k \geq 0 y_{k}=f^{k}\left(x_{n+k}\right) .
$$

Definition 1.9. - A puzzle $V$ is determined if:

$$
u, v \succeq_{f}^{1} w \text { and } i_{1}(u)=i_{1}(v) \Longrightarrow u=v \text {. }
$$

Remarks.

(1) Many puzzles are determined, including: those defined by subshifts and those defined as in Section 1.2 under the extra assumptions: (i) $T$ is one-to-one on the closure of each element of $\mathcal{P}$; (ii) for each $x \in M$, $\lim _{n \rightarrow \infty} \operatorname{diam}\left(\mathcal{P}^{n}(x)\right)=0$ where $\mathcal{P}^{n}(x)$ is the unique element (if it exists) of $\mathcal{P}^{n}$ that contains $x$.

(2) There exist determined puzzles whose duals are not determined.

QFT subshifts are determined QFT puzzles. - Let $(\sigma, X)$ be an arbitrary QFT subshift. Let $V$ be the puzzle defined by $X$ as in section 1.2. As remarked above, $V$ is determined. Also, subshifts being expansive, $h(\sigma, \mu)=h\left(\sigma, \mu, \epsilon_{0}\right)$ for some $\epsilon_{0}>0$ depending only on the choice of the metric, not on $\mu$, so the W-local entropy is zero. As remarked after Definition 1.4 the irreducible pieces of $V$ can be identified with the minimum left constraints of $X$. Hence the constraint entropies of the puzzle $V$ and of the subshift $X$ are the same. Thus

$$
h_{\mathcal{C}}(V)+h_{\mathrm{wloc}}(V)=h_{\mathcal{C}}(X)<h_{\mathrm{top}}(V)=h_{\mathrm{top}}(V),
$$

proving the claim.

1.4. Structure Theorem. - Let us first recall the notion of entropy-conjugacy from 6 .

Let $T: X \rightarrow X$ be a Borel map. Let $h(T)$ be the supremum of the entropy of all $T$-invariant probability measures. Recall that if $X$ is compact and $T$ continuous then $h(T)$ is just the topological entropy by the variational principle [39. A subset $X_{0} \subset X$ is entropy-negligible if it is Borel and satisfies:

$$
\tilde{h}\left(T, X_{0}\right):=\sup \left\{h(T, \mu): \mu \text { ergodic with } \mu\left(X_{0}\right)>0\right\}<h(T),
$$

that is, $X_{0}$ is negligible in the usual sense for all large entropy measures, i.e., invariant and ergodic probability measures with entropy close to $h(T)$.

Two Borel maps $T: X \rightarrow X$ and $S: Y \rightarrow Y$ are entropy-conjugate ${ }^{(1)}$ if there exist entropy-negligible subsets $X_{0} \subset X$ and $Y_{0} \subset Y$ and a Borel isomorphism $\Psi: X \backslash X_{0} \rightarrow Y \backslash Y_{0}$ with $\Psi \circ T=S \circ \Psi$. The constant of this isomorphism is $\max \left(\tilde{h}\left(T, X_{0}\right), \tilde{h}\left(S, Y_{0}\right)\right)$.

(1) The name entropy-conjugacy was introduced by Bowen 4 for a similar notion: topological conjugacy after discarding subsets having small dimension-like entropy. 
Recall also that a Markov shift (see [17] and also [13, 24, 26, 34, 35, 36, for background) is the set $\Sigma(G)$ of all bi-infinite paths on a countable or finite directed graph $G$ together with the left-shift $\sigma$ :

$$
\Sigma(G)=\left\{x \in G^{\mathbb{Z}}: \forall n \in \mathbb{Z} x_{n} \rightarrow x_{n+1} \text { on } G\right\} \text { and } \sigma\left(\left(x_{n}\right)_{n \in \mathbb{Z}}\right)=\left(x_{n+1}\right)_{n \in \mathbb{Z}} .
$$

The Markov shifts defined by finite graphs are the classical subshifts of finite type (of order 1) - see 25. for an introduction to this rich classical theory.

$\Sigma(G)$ is irreducible if it contains a dense orbit (equivalently $G$ is strongly connected). Any Markov shift has a spectral decomposition as a union of countably many irreducible Markov subshifts (up to wandering orbits). The period of a subset $U$ of $\Sigma(G)$ is the greatest common divisor of all $k \geq 1$ such that $\sigma^{k} U \cap U \neq \emptyset$. The period of $\Sigma(G)$ is the largest period of all non-empty open subsets of $\Sigma(G)$.

A Markov shift is not compact unless it is a subshift of finite type. Its topological entropy is therefore defined as explained above for a general Borel system. Gurevič 15. proved that in the irreducible case this entropy is just, for any $(a, b) \in G^{2}$ :

$$
h(G):=h(\Sigma(G))=\limsup _{n \rightarrow \infty} \frac{1}{n} \log \#\left\{v \in G^{n}: v_{1}=a, v_{1} \rightarrow v_{2} \rightarrow \ldots v_{n}=b\right\} .
$$

An irreducible Markov shift is said to be SPR (for stably positively recurrent [17]also called strongly positively recurrent [37]) if it admits an entropy-maximizing probability measure $\mu$ which is exponentially filling, i.e., for any non-empty open subset $U$ of $X$,

$$
\lim _{n \rightarrow \infty} \frac{1}{n} \log \mu\left(X \backslash \bigcup_{k=0}^{n} \sigma^{-n} U\right)<0 .
$$

Such Markov shifts are closest to being of finite type by a number of results (see, e.g., Gurevič 16, Sarig [35, Gurevič-Savchenko 17] among others).

In Sec. 4.1 we shall associate to any puzzle a Markov shift $\Sigma(\mathcal{D})$ defined by the adaptation to puzzles of the "complete" Hofbauer diagram developed in [6] for subshifts $(2)$

Finally recall that the natural extension of a map $T: X \rightarrow X$ is the "smallest" extension that is invertible, i.e., it is $\tilde{T}: \tilde{X} \rightarrow \tilde{X}$ with $\tilde{X}:=\left\{x \in X^{\mathbb{Z}}: \forall n \in \mathbb{Z}\right.$ $\left.T\left(x_{n}\right)=x_{n+1}\right\}$ and $\tilde{T}\left(\left(x_{n}\right)_{n \in \mathbb{Z}}\right)=\left(T x_{n}\right)_{n \in \mathbb{Z}}$.

We may now state our key structure theorem:

Theorem 1 (Main Result). - Let $V$ be a puzzle. Let $\mathcal{D}$ be its complete Markov diagram, defined in Sec. 4.1 below.

(1) If $V$ is $*-Q F T$, the natural extension of the dynamics of $V$ is entropy-conjugate with constant at most $h_{\mathcal{C}}(V)$ to the Markov shift $\Sigma(\mathcal{D})$.

(2) If $V$ is $Q F T$, then, for every $H>h_{\mathcal{C}}(V)+h_{\text {wloc }}(V)$, the spectral decomposition of $\Sigma(\mathcal{D})$ contains only finitely many irreducible Markov shifts with entropy $\geq H$.

(2) This "complete" variant essentially removes "accidental" identifications, i.e., of the type $T(A)=$ $T(B)$ where $A$ and $B$ are distinct elements of the partition whereas $T(A)=T(B)$ does not belong to that partition. This variant is necessary for the precise counting of periodic orbits as we explained in 11] (it also simplifies the proof of the partial isomorphism, see Section 4). 
Moreover these Markov shifts are SPR. More precisely their entropies at infinity (see Definition 1.10 below) are at most $h_{\mathcal{C}}(V)+h_{\mathrm{wloc}}(V)$.

(3) If $V$ is both $Q F T$ and determined then, for any $\epsilon>0$, there are an integer $N$ and a finite part $\mathcal{D}_{*} \subset \mathcal{D}$ such that the following property holds.

There is a period-preserving bijection between the periodic loops on $\mathcal{D}$ that meet $\mathcal{D}_{*}$ and the periodic orbits of $i_{N}\left(X_{V}\right)$ after discarding a number $p_{n}^{0}$ of the n-periodic orbits of $i_{N}\left(X_{V}\right)$ satisfying:

$$
\limsup _{n \rightarrow \infty} \frac{1}{n} \log p_{n}^{0} \leq h_{\mathcal{C}}(V)+h_{\text {wloc }}(V)+\epsilon .
$$

The proof of this theorem is presented in Sections 4 and 5 .

\subsection{Dynamical consequences. -}

1.5.1. Maximum measures. - The Structure Theorem gives the following, using Gurevič's result on maximum measures for Markov shifts. Recall that a Bernoulli scheme is the shift $\sigma$ acting on the set of sequences $\{1, \ldots, s\}^{\mathbb{Z}}$ ( $s$ a positive integer) endowed with the invariant and ergodic probability measure $\mu$ defined by:

$$
\mu\left(\left\{\alpha: \alpha_{0} \ldots \alpha_{k}=a_{0} \ldots a_{k}\right\}\right)=p\left(a_{0}\right) \ldots p\left(a_{n}\right)
$$

where $(p(1), \ldots, p(s))$ is a probability vector. A finite extension is the product $(X, \sigma)$ with a permutation on a finite set.

For the sake of brevity, a maximum measure will be any ergodic, invariant probability measure with maximum entropy.

Theorem 2 (Maximum Measures). - A QFT puzzle has at least one and at most finitely many maximum measure.

More precisely, those are in bijection with the SPR Markov subshifts with maximum entropy and the natural extensions of these measures are measure-preservingly isomorphic to finite extensions of Bernoulli schemes,

Moreover, the periods of the (cyclic permutations of the) measures and those of the irreducible subshifts coincide.

This follows from the Structure Theorem and Gurevič results for Markov shifts, as explained in Section 7.1 .

Remark. The proof that the QFT condition implies the existence of a maximum measure is closely related to a joint work $\mathbf{1 2}$ with S. Ruette.

1.5.2. Zeta functions. - We turn to the numbers of periodic points.

Theorem 3 (Zeta Functions). - Assume that $V$ is a QFT puzzle which is also determined. Fix a large integer $N$ and consider the reduced zeta function:

$$
\zeta_{N}(z):=\exp \sum_{n \geq 1} \frac{z^{n}}{n} \#\left\{x \in i_{N}\left(X_{V}\right): \sigma^{n}(x)=x\right\} .
$$


$\zeta_{N}$ is holomorphic on $|z|<e^{-h_{\mathrm{top}}(V)}$ and has a meromorphic extension to $|z|<$ $e^{-h_{\mathcal{C}}(V)-h_{\mathrm{wloc}}(V)}$. Its singularities near the circle $|z|=e^{-h_{\mathrm{top}}(V)}$ are exactly poles at

$$
e^{2 i \pi k / p_{i}} e^{-h_{\mathrm{top}}(V)} \quad i=1, \ldots, r \quad k=0, \ldots, p_{i}-1
$$

(with multiplicities equal to repetitions in this list) where $p_{1}, \ldots, p_{r}$ are the periods of the distinct maximum measures $\mu_{1}, \ldots, \mu_{r}$.

Moreover, for each $\epsilon>0$, the poles of $\zeta_{N}(z)$ in $|z|<e^{-h_{\mathcal{C}}(V)-h_{\mathrm{wloc}}(V)-\epsilon}$ are independent of $N$ : for $N^{\prime}, N>N(V, \epsilon), \zeta_{N^{\prime}}(z) / \zeta_{N}(z)$ extends to a holomorphic function on this disk.

This is, technically, the most delicate result as we have to go from entropy estimates (which confuses very close points) to counting (this is of course why the determinacy assumption is required) - see Section 7.2 .

Remark.

1. In contrast to [1], the lower-bound on the meromorphy radius will be obtained using a new result about general Markov shifts.

2. Counting the projections at level $N$ of periodic points instead of the periodic points themselves is necessary as it not even true that $\#\left\{x \in X_{V}: \sigma^{n}(x)=x\right\}<\infty$ for any determined QFT puzzle - see Section 2.3.

1.5.3. Semi-local zeta functions for SPR Markov shifts. - The proof of Theorem 3 relies on a similar (and new) result for SPR Markov shifts. First, define the "entropy at infinity":

Definition 1.10. - Let $G$ be a countable, oriented, irreducible graph. The entropy at infinity of $G$ is:

$$
h_{\infty}(G)=\inf _{F \subset \subset G} \inf _{\mu_{0}>0} \sup \left\{h(\sigma, \mu): \mu([F])<\mu_{0}\right\}
$$

where $F$ ranges over the finite subgraphs of $G$ and $[F]:=\left\{x \in \Sigma(G): x_{0} \in F\right\}$.

Remarks. (1) $h_{\infty}(G)=-\infty$ if $G$ is finite.

(2) $H \subset G$ implies that $h_{\infty}(H) \leq h_{\infty}(G)$ as both are infimum over $\mu_{0}>0$ and $F \subset \subset G$ of $\sup \left\{h(\sigma, \mu): \mu([F])<\mu_{0}\right\}$ and $\sup \left\{h(\sigma, \mu): \mu([F])<\mu_{0}\right.$ and $\mu([G \backslash H])=0\}$, respectively.

(3) This definition was motivated by the observation of Ruette 34 that the combinatorial quantities considered by Gurevič and Zargaryan 18 were related to entropy at infinity. In particular, $h_{\infty}(G)<h(G)$ iff $G$ is SPR (see Proposition 6.1).

Theorem 4. - Let $\Sigma(G)$ be an irreducible Markov shift with finite Gurevic entropy $h(G)$. For any finite subset $F \subset \subset G$, the semi-local zeta function of $G$ at $F$ :

$$
\zeta_{F}^{G}(z):=\exp \sum_{n \geq 1} \frac{z^{n}}{n} \#\left\{x \in \Sigma(G): \sigma^{n}(x)=x \text { and }\left\{x_{0}, \ldots, x_{n-1}\right\} \cap F \neq \emptyset\right\}
$$

is holomorphic on $|z|<e^{-h(G)}$ and has a meromorphic extension to $|z|<e^{-h_{\infty}(G)}$. 
Moreover, for every $\epsilon>0$, there exists $F_{0} \subset \subset G$ such that, if $F, F^{\prime}$ are two finite subsets with $F_{0} \subset F, F^{\prime} \subset \subset G$, then

$$
\frac{\zeta_{F^{\prime}}^{G}(z)}{\zeta_{F}^{G}(z)} \text { is holomorphic and non-zero on }|z|<e^{-\left(h_{\infty}(G)+\epsilon\right)} \text {. }
$$

Remarks.

(0) Notice that the semi-local zeta functions at a single vertex coincide with the local zeta functions of [17] but differ from those of [36] (which have usually a nonpolar singularity at $z=e^{-h(G)}$ so has no meromorphic extension).

(1) This result is new. In fact, even the case of where $F$ is reduced to a single vertex had not been observed to our knowledge.

(2) The theorem is trivial if $h_{\infty}(G)=h(G)$, that is, if $G$ is not SPR (see Proposition 6.1). In the opposite extreme, for subshifts of finite type, i.e., $G$ finite, this asserts that $\zeta_{F}^{G}$ extends meromorphically over $\mathbb{C}$. Of course, in this case $\zeta_{F}^{G}=\zeta^{G} / \zeta^{G \backslash F}$ in terms of the classical Artin-Mazur zeta functions so the semi-local zeta function extends in fact meromorphically over the Riemann sphere, i.e., is a rational function.

(3) The conclusion of Theorem 4 is false for the full zeta function (i.e., $\zeta^{G}$ ). $\zeta^{G}$ is not always defined as a formal series and, even if it is, can have zero radius of convergence or it can have various types of singularities (see [17, Example 9.7]).

(4) For two finite subsets $F, H, \zeta_{F}^{G}(z) / \zeta_{H}^{G}(z)$ is meromorphic over $|z|<\exp -h_{\infty}(G)$ but it is not necessarily holomorphic and non-zero. If $G_{n}$ is the complete oriented graph on $\{1,2, \ldots, n\}$, we have $h\left(G_{3}\right)=\log 3, h_{\infty}\left(G_{3}\right)=-\infty, \zeta^{G_{3}}(z)=\zeta_{G_{3}}^{G_{3}}(z)=$ $1 /(1-3 z)$ and $\zeta_{\{0\}}^{G_{3}}(z)=\zeta^{G_{3}}(z) / \zeta^{G_{2}}(z)=(1-2 z) /(1-3 z)$.

(5) The maximum radius of a meromorphic extension $3(3)$ of the semi-local zeta functions may be strictly larger than $\exp -h_{\infty}(G)$. Indeed, there are Markov shifts for which the radius of meromorphy of the local zeta functions varies (see Appendix A for an example where some local zeta functions are rational and others have a finite radius of meromorphy). One can wonder if these values and for instance their supremum have a dynamical significance besides the obvious fact that if $g \in G$ and $g^{\prime} \in G^{\prime}$ define the same local zeta functions, the corresponding shifts are almost isomorphic in the sense of $[\mathbf{3}$. One would like to "patch together" all the partial informations provided by all the (semi) local zeta functions.

The proof of Theorem 4 relies on the generalization of an algebraic formula decomposing the determinant of finite matrices - see section [6. In the special case of a loop graph, i.e., the disjoint union of $f_{n}$ loops for each length $n \geq 1$ based at a single vertex $a$ (see Appendix A), with $F$ reduced to $\{a\}, h_{\infty}(G)=\lim \sup _{n \geq \infty}(1 / n) \log f_{n}$ and the determinantal formula coincides with the well-known identity $\zeta_{a}(f)=\left(1-f_{a}(z)\right)^{-1}$, where $f_{a}(z):=\sum_{n \geq 1} f_{n} z^{n}$, the rest of the proof following then that of [17. Prop. 9.2].

1.5.4. Equidistribution of the periodic points. - The periodic points are equidistributed w.r.t. a suitable measure of maximum entropy:

${ }^{(3)}$ See Appendix A for formal definitions. 
Theorem 5 (Equidistribution of periodic points). - Assume that $V$ is a QFT puzzle which is determined. Let $\mu_{1}, \ldots, \mu_{r}$ be the distinct maximum measures and $p_{1}, \ldots, p_{r}$ their periods, $p=\operatorname{lcm}\left(p_{1}, \ldots, p_{r}\right)$.

Fix a sufficiently large integer $N$ and consider, for $n \in p \mathbb{Z}$, the measures:

$$
\mu_{n}^{N}:=\sum_{x \in i_{N}\left(X_{V}\right) \mid \sigma^{n}(x)=x} \delta_{x} .
$$

Then, in the weak star topology,

$$
\lim _{n \rightarrow \infty, n \in p \mathbb{Z}} \frac{1}{\mu_{n}^{N}\left(X_{V}\right)} \mu_{n}^{N}=\frac{1}{\sum_{i} p_{i}} \sum_{i=1}^{r} p_{i} \mu_{i} .
$$

This will also be a consequence of a result of Gurevič and Savchenko [17] for SPR Markov shifts.

1.6. Classification of QFT puzzles. - In the same way as QFT subshifts 11, QFT puzzles can be classified up to entropy-conjugacy by their entropy and periods. Using the classification result [3] obtained with Boyle and Gomez for SPR Markov shifts, Theorem 11implies:

Theorem 6 (Classification). - The natural extension of QFT puzzles are completely classified up to entropy-conjugacy by the following data: the topological entropy and the list, with multiplicities, of the periods of the finitely many maximum measures.

This gives a very precise meaning to our assertion that complexity assumptions (defining QFT puzzles) in fact characterize them from the point of view of complexity.

1.7. Smooth maps defining QFT puzzles. - We describe the class of smooth maps whose symbolic dynamics are QFT puzzles which will both provide interesting examples of such puzzles and yield a new proof of variants of previous results 9 ] about the dynamics of such maps.

Entropy-expansion. - Let $F: M \rightarrow M$ be a $C^{\infty}$ smooth map of a $d$-dimensional compact manifold. The main assumption is that $F$ is entropy-expanding, which is defined as follows. The codimension one entropy $\mathbf{9}]$ is

$$
h^{d-1}(F)=\sup \left\{h_{\text {top }}\left(F, \phi\left([0,1]^{d-1}\right): \phi \in C^{\infty}\left(\mathbb{R}^{d-1}, M\right)\right\} .\right.
$$

Recall that $h_{\mathrm{top}}(F, \sigma)$ counts the number of orbits starting from the not necessarily invariant set $\sigma$ - see [39]:

$$
h_{\mathrm{top}}(F, \sigma)=\lim _{\epsilon \rightarrow 0} \limsup _{n \rightarrow \infty} r(\epsilon, n, \sigma) .
$$

The entropy-expanding condition [9] is:

$$
h^{d-1}(F)<h_{\text {top }}(F):=h_{\text {top }}(F, M) .
$$

It is an open condition in the $C^{\infty}$ topology [9]. Entropy-expanding maps form a natural class of multi-dimensional non-uniformly expanding maps. This class includes all couplings of interval maps, e.g., all self-maps of $[0,1]^{2}$ of the form:

$$
(x, y) \mapsto(a x(1-x)+\epsilon y, b y(1-y)+\epsilon x)
$$


for $3.569 \ldots<a, b<4(3.569 \ldots$ is the Feigenbaum parameter $)$.

Indeed, $x \mapsto t x(1-x)$ maps $[0,1]$ into $[0, t / 4]$ for $0 \leq t \leq 4$ and has positive entropy for $t>3.569 \ldots$, so that for $\epsilon=0$, the above is entropy-expanding by [7]. For $\epsilon \geq 0$ small enough, the coupling (4) still preserves $[0,1]^{2}$. This coupling is finally entropy-expanding by the openness of this condition.

Such coupled interval maps are natural examples of multi-dimensional non-uniformly expanding maps with critical points but their ergodic theory has resisted all other approaches up to now, despite all the results following [38] in the case where one of the two factors is assumed to be uniformly expanding.

Good partitions. - We shall additionaly assume that there exists a good partition $\mathcal{P}$ for $F$, i.e., with the following properties:

$-\mathcal{P}$ is finite;

- each element of $\mathcal{P}$ is the closure of its interior;

- the boundary of each element of $\mathcal{P}$ is the image of a compact subset of $\mathbb{R}^{d-1}$ by a $C^{\infty}$ smooth map;

- the restriction of $f$ to the closure of any element $A$ of $\mathcal{P}, f \mid \bar{A}$, is one-to-one.

- for each $n \geq 1$, each $\mathcal{P}, n$-cylinder:

$$
A_{0} \cap F^{-1} A_{1} \cap \cdots \cap F^{-n+1} A_{n-1} \quad A_{i} \in \mathcal{P}
$$

has only finitely many almost connected components: maximum subsets which cannot be split into two subsets at a positive distance;

- we have a uniform bound

$$
\sup _{x \in M} \#\left\{k \in \mathbb{N}: F^{k}(x) \in \partial \mathcal{P}\right\}<\infty .
$$

There are many $C^{\infty}$ maps of compact manifolds which fail to have a good partition. Indeed, it is not difficult to construct $C^{\infty}$ entropy-expanding maps which are boundedto-one on no open and dense set.

On the other hand we believe that among $C^{\infty}$ maps, having a good partition is generic, i.e., this property defines a subset which contains a countable intersection of open and dense subsets. As a step in this direction, we prove the following in Appendix B

Proposition 1.11. - The coupling in eq. (4) has a good partition for all parameters $(a, b, \epsilon)$ except for at most a countable union of smooth hypersurfaces in $\mathbb{R}^{3}$.

Puzzles of Good Entropy-Expanding Maps. — Given an entropy-expanding $C^{\infty}$ smooth map $F$ with a good partition $\mathcal{P}$ as above, we define the associated puzzle to be $(V, i, f)$ with $V=\sqcup_{n \geq 0} V_{n}$ where:

$-V_{n}$ is the collection of almost connected components of $\mathcal{P}, n$-cylinders;

$-i, f: V_{n+1} \rightarrow V_{n}$ are the maps defined by $i(u)=v$ and $f(u)=w$ if $v \supset u$ and $w \supset F(u)$;

(this is a special case of the construction given in section 1.2). We shall see the: 
Theorem 7 (Puzzles of entropy-expanding maps). - Let $T: M \rightarrow M$ be a $C^{\infty}$ smooth map of a d-dimensional compact manifold. Assume that $T$ is entropyexpanding and admits a good partition $\mathcal{P}$. Then the puzzle associated to $(T, \mathcal{P})$ is QFT and determined. More precisely, $h_{\mathrm{wloc}}(V)=0$ and $h_{\mathcal{C}}(V) \leq h^{d-1}(T)$.

In particular, such maps have finitely many maximum measures and, up to the identifications given by some partition, their periodic points define zeta functions with meromorphic extensions to $|z|<\exp -h^{d-1}(T)$.

These results are stated more precisely as Theorem 8 and its Corollaries 8.28 .3 , Remark. One can relax the assumption of smoothness to $C^{r}$ smoothness with $r \geq 1$ provided one strengthens the entropy-expansion condition in the following way:

$$
h^{d-1}(f)+\frac{d-1}{r} \log ^{+} \operatorname{Lip}(f)<h_{\mathrm{top}}(f)
$$

and using that, according to $\left[\mathbf{6}\right.$, the left hand side dominates $H^{d-1}(f)$, the uniform codimension 1 entropy defined in that work. We thus obtain a new existence result. It generalizes the classical result of existence of a maximum measure for all piecewise monotone maps (i.e., $f:[0,1] \rightarrow[0,1]$ such that $[0,1]=\bigcup_{i=1}^{N}\left[a_{i}, b_{i}\right]$ with $f \mid\left(a_{i}, b_{i}\right)$ is continuous and strictly monotone) with positive topological entropy.

Numbering. All items are numbered consecutively within each section, except for the theorems.

Acknowledgments. J.-C. Yoccoz asked me about the relationship between Hofbauer's towers and the puzzles of complex dynamics a long time ago. M. Boyle was always encouraging at all the stages this work went through. S. Ruette pointed out to me Proposition 6.1 and the link with $h_{\infty}(G)$. I am indebted to O. Sarig, especially with regard to my results about zeta functions for Markov shifts. His insights led me to the example given in the Appendix.

The observations of the referees have also significantly improved the exposition of this paper (including section 6).

\section{Further Examples}

2.1. Puzzles with finitely many irreducible vertices. - We prove Lemma 1.5, i.e., that the dynamics of a puzzle with finitely many irreducible vertices is (topologically conjugate to) a subshift of finite type.

Let $n_{0}$ be the largest integer such that $V_{n_{0}}$ contains a $f$-irreducible piece. Let $n$ be an arbitrary integer larger than $n_{0}$. Recall the map $i_{n}: X_{V} \rightarrow V_{n}^{\mathbb{N}}$ from (2). To prove the lemma, it is enough to see that $i_{n}\left(X_{V}\right)$ is a subshift of finite type (easy since $v$ reducible implies $\left.F_{V}\left([v]_{V}\right)=[f(v)]_{V}=\bigcup_{w \in i^{-1}(v)}[w]_{V}\right)$ and that all the subshifts obtained for large $n$ are topologically conjugate by the maps induced by the restrictions $i: V_{m} \rightarrow V_{n}, m \geq n$.

Let $n>n_{0}$. Consider the finite graph $\Gamma_{n}$ whose vertices are the elements of $V_{n}$ and whose arrows are defined by:

$$
u \rightarrow \Gamma_{n} v \Longleftrightarrow \exists w \in V_{n+1} i(w)=u \text { and } f(w)=v .
$$




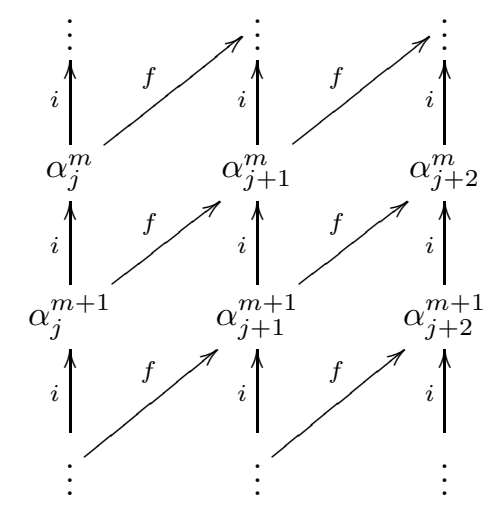

Figure 1. Construction of $\alpha^{m+1}$.

Observe for future reference that, because of the definition of a reducible vertex, $w$ above is uniquely determined by $u$ and $v$. $\Sigma_{n}$.

Let $\Sigma_{n} \subset V_{n}^{\mathbb{N}}$ be the subshift of finite type defined by $\Gamma_{n}$. We claim that $i_{n}\left(X_{V}\right)=$

Observe first that $i_{n}\left(X_{V}\right) \subset \Sigma_{n}$. Indeed, for $x \in X_{V}$ and $k \geq 0, i\left(\left(F_{V}^{k} x\right)_{n+1}\right)=$ $\left(F_{V}^{k} x\right)_{n}$ by definition of $X_{V}$ and $f\left(\left(F_{V}^{k} x\right)_{n+1}\right)=\left(F_{V}^{k+1} x\right)_{n}$ by definition of $F_{V}$. Thus, $\left(F_{V}^{k} x\right)_{n} \rightarrow_{\Gamma_{n}}\left(F_{V}^{k+1} x\right)_{n}$, and $i_{n}(x) \in \Sigma_{n}$.

We turn to the converse inclusion. Let $\alpha^{0} \in \Sigma_{n}$ for some $n>n_{0}$. We are going to define inductively $\alpha^{m} \in V_{n+m}^{\mathbb{N}}, m \geq 1$, such that, for all $m \geq 0, j \in \mathbb{N}$ and $0 \leq k \leq m$,

$$
\text { (i) } i^{k}\left(\alpha_{j}^{m}\right)=\alpha_{j}^{m-k} \text {, (ii) } \alpha_{j}^{m} \succeq_{f}^{k} \alpha_{j+k}^{m-k} \text {, (iii) } \alpha_{j+1}^{m} \in f\left(i^{-1}\left(\alpha_{j}^{m}\right)\right) \text {. }
$$

This will imply that $i_{n}\left(X_{V}\right) \supset \Sigma_{n}$. Indeed, recall that $x=i_{n}\left(\alpha^{0}\right)$ means that $x_{k}=i^{n-k}\left(\alpha_{0}^{0}\right)$ for $k \leq n$ and $x_{k}=\alpha_{0}^{k-n}$ for $k \geq n$. Hence $x \in X_{V}$ by (i) and $i_{n}(x)=\alpha^{0}$, as $f^{k}\left(\alpha_{0}^{k}\right)=\alpha_{k}^{0}$ by (ii).

Observe that (5) holds for $m=0$ because of the definition of $\Gamma_{n}$. Let us assume that $\alpha_{j}^{p}$ has been defined for $p \leq m$ and all $j \in \mathbb{N}$ so that eq. (5) is satisfied. For $j \in \mathbb{N}$, let us build $\alpha_{j}^{m+1}$ satisfying (5).

Let $\alpha_{j}^{m+1} \in f^{-1}\left(\alpha_{j+1}^{m}\right) \cap i^{-1}\left(\alpha_{j}^{m}\right)$ (this intersection is not empty by (iii), eq. (5) and it is unique because $\alpha_{j}^{m+1}$ is $f$-reducible). Let us check eq. (5) for $m+1, j$. $i^{k+1}\left(\alpha_{j}^{m+1}\right)=i^{k}\left(\alpha_{j}^{m}\right)$ hence (i) is satisfied. $f\left(\alpha_{j}^{m+1}\right)=\alpha_{j+1}^{m}$ and $\alpha_{j}^{m+1}$ is $f$-reducible by the main assumption. Thus $\alpha_{j}^{m+1} \succeq_{f}^{k} \alpha_{j+k}^{m+1-k}$ for $k=1$ and for $1<k \leq m$ by the induction hypothesis. This is (ii). As $\alpha_{j}^{m+1} \succeq_{f}^{1} \alpha_{j}^{m}$, the $i$-tree below $\alpha_{j}^{m+1}$ is mapped by $f$ onto the $i$-tree below $\alpha_{j}^{m}$. This gives (iii), completing the induction.

Finally, one observes that $\alpha^{p+1}=i_{p+1}(\underline{x})$ is uniquely defined by $\alpha^{p}=i_{p}(\underline{x})$ so that the natural projection $i_{p+1}\left(X_{V}\right) \rightarrow i_{p}\left(X_{V}\right)$ is in fact a homeomorphism. This finishes the proof of Lemma 1.5 . 
2.2. *-QFT puzzles with nasty dynamics. - We give examples of *-QFT puzzles with infinitely or no maximum measures.

Let $\Sigma_{0}=\left\{0^{\infty}\right\}, \Sigma_{1}, \Sigma_{2}, \ldots$ be a sequence of subshifts of finite type over disjoint alphabets. Assume that the Markov order of $\Sigma_{n}$ is at most $n$ (i.e., $A \in \Sigma_{n}$ iff $A_{k} \ldots A_{k+n-1}$ is a word in $\Sigma_{n}$ for all $\left.k \geq 0\right)$ and that $h_{\text {top }}\left(\Sigma_{n}\right)>0$ for all $n \geq 1$. We are going to build a puzzle which is conjugate to $\bigcup_{n \geq 0} \Sigma_{n}$. Taking $h_{\text {top }}\left(\Sigma_{n}\right)=\log 2$ for all $n \geq 1$, or $h_{\text {top }}\left(\Sigma_{n}\right) \nearrow \log 2$ as $n \rightarrow \infty$, shall yield the required examples.

Let $L_{n}\left(\Sigma_{k}\right)$ be the set of words of length $n$ appearing in $\Sigma_{k}$. The puzzle will be $(V, i, f)$ defined as follows:

Let $V_{0}=\{\emptyset\}$ (the empty word) and $V_{n}=\sqcup_{0 \leq k \leq n} L_{n}\left(\Sigma_{k}\right)$.

Let $w:=A_{1} \ldots A_{n} \in V_{n}$. If $w \in L_{n}\left(\Sigma_{n}\right)$, then $f(w)=i(w)=0^{n-1}$. Otherwise, let $f\left(A_{1} \ldots A_{n}\right)=A_{2} \ldots A_{n}$ and $i\left(A_{1} \ldots A_{n}\right)=A_{1} \ldots A_{n-1}$.

The only vertices of $V_{n}$ that can be irreducible are those $w \in L_{n}\left(\Sigma_{n}\right)$ which are mapped by $f$ to $0^{n-1}$. For $n>N$, all these vertices are confused with $0^{n}$ by $i_{N}$. Thus at a given level $N$, the number of distinguishable irreducible vertices in $V_{n}$ is bounded independently of $n$ so that $h_{\mathcal{C}}(V)=0$. Thus $V$ is indeed a $*$-QFT puzzle.

2.3. QFT puzzles with bad zeta functions. - Let us describe a determined QFT puzzle with infinitely many periodic orbits of any given length so that the zeta function defined from the periodic points (and not their projections) is not even welldefined as a formal series.

Pick a sequence of positive integers $p_{1}, p_{2}, \ldots$ such that $\# p^{-1}(k)=\infty$ for all $k \geq 1$. Modify the previous construction taking $\Sigma_{0}:=\{0,1\}^{2}$ and, for $n \geq 1$, $\Sigma_{n}:=\left\{\sigma^{j} \omega^{n}: 0 \leq j<p_{n}\right\}$, a periodic orbit of length $p_{n} \geq 1$. Take the symbols $\left(\omega_{j}^{n}\right)_{n \geq 1,0 \leq j<p_{n}}$ pairwise distinct and disjoint from $\{0,1\}$.

Observe that, for any word $w$ from some $\Sigma_{n}, n \geq 1, \mathcal{T}_{i}(w)$ is a linear graph whereas $\mathcal{T}_{i}(w)$ for all the other words are not linear. It follows that the irreducible pieces are: (i) the one-letter words 0 and 1; (ii) the words from $\Sigma_{n}$ of length $n$. Thus, $h_{\mathcal{C}}(V)=h_{\text {wloc }}(V)=0$.

Let $u, u^{\prime}$ be words, $u$ not from $\Sigma_{0}$ such that $u, u^{\prime} \succeq_{f}^{1} w$ and $i_{1}(u)=i_{1}\left(u^{\prime}\right)$. $\mathcal{T}_{i}(u)$ is then a linear graph forcing $\mathcal{T}_{i}\left(u^{\prime}\right)$ to be so. It follows that $u=\omega_{j}^{n} \ldots \omega_{j+\ell}^{n}$ and $u^{\prime}=\omega_{k}^{m} \ldots \omega_{k+\ell}^{m}$ for some integers $j, k, \ell, n, m$ with $\ell \geq 1$ by reducibility. Hence $f(u)=f\left(u^{\prime}\right)$ implies that $\omega_{j+1}^{n}=\omega_{k+1}^{m}$. By the choice of pairwise distinct symbols, this yields $u=u^{\prime}: V$ is determined.

Therefore $V$ is indeed a determined QFT puzzle.

Remark. Obvious adaptations of this construction yield examples with arbitrary growth rates of the number of periodic orbits.

\section{Basic properties}

\subsection{Some properties of $f$-reducibility. -}


Lemma 3.1. - If $i(u)=i\left(u^{\prime}\right), f^{k}(u)=f^{k}\left(u^{\prime}\right)$ and $u \succeq_{f}^{k} v$ and $f: \mathcal{T}_{i}\left(f^{l-1}\left(u^{\prime}\right)\right) \rightarrow$ $\mathcal{T}_{i}\left(f^{l}\left(u^{\prime}\right)\right), l=1, \ldots, k$ are graph isomorphisms then $u=u^{\prime}$. In particular,

$$
i(u)=i\left(u^{\prime}\right) \text { and } u \succeq_{f}^{k} w \text { and } u^{\prime} \succeq_{f}^{l} w \Longrightarrow k=l \text { and } u=u^{\prime} .
$$

Proof: (6) clearly follows from the first claim. For $k=0,1$, this claim follows from the definition of $\succeq_{f}^{k}$. Assume the claim for some $k-1 \geq 0$ and let $u, u^{\prime}$ and $v$ be as in the claim for $k$. Now, $i\left(f^{k-1}(u)\right)=f^{k-1}(i(u))=f^{k-1}\left(i\left(u^{\prime}\right)\right)=i\left(f^{k-1}\left(u^{\prime}\right)\right)$ and both $f^{k-1}(u) \succeq_{f}^{1} v$ and $f: \mathcal{T}_{i}\left(f^{k-1}\left(u^{\prime}\right)\right) \rightarrow \mathcal{T}_{i}(v)$ is an isomorphism. This implies that $f^{k-1}(u)=f^{k-1}\left(u^{\prime}\right)=: w$ by the definition of $\succeq_{f}^{1}$. Now $i(u)=i\left(u^{\prime}\right)$ and $u \succeq_{f}^{k-1} w$ and $f: \mathcal{T}_{i}\left(f^{l-1}\left(u^{\prime}\right)\right) \rightarrow \mathcal{T}_{i}\left(f^{l}\left(u^{\prime}\right)\right), l=1, \ldots, k-1$, so the induction hypothesis implies $u=u^{\prime}$.

Lemma 3.2. - If $i(u) \succeq_{f}^{k} i(v)$ and $f^{k}(u)=v$ with $|u|,|v| \geq 1$ and $k \geq 0$, then $u \succeq_{f}^{k} v$. In particular, if $u$ with $|u|>1$ is $f$-irreducible, then so is $i(u)$.

ProOF: $i(u) \succeq_{f}^{k} i(v)$ implies that the $i$-trees below $i(u)$ and $i(v)$ are isomorphic through $f^{k}$. This implies the same for the sub- $i$-trees below $u$ and $v$. Assuming by contradiction that $u \nsucceq_{f}^{k} v$ we obtain that there exists $w \in i^{-1}(i(u)), w \neq u$ with $f^{k}(w)=f^{k}(u)$, but this would contradict that $f^{k} \mid \mathcal{T}_{i}(i(u))$ is one-to-one.

3.2. Natural extension and duality. - Except in trivial cases, the dynamics $F_{V}: X_{V} \rightarrow X_{V}$ is non-invertible. To obtain an invertible dynamical system, one goes to the natural extension. It can be described as $\left(\underline{X}_{V}, F_{V}\right)$ with:

$$
\begin{gathered}
\underline{X}_{V}=\left\{\left(v_{n, p}\right)_{n, p}: \forall(n, p) \in \mathbb{N} \times \mathbb{Z} i\left(v_{n+1, p}\right)=f\left(v_{n+1, p-1}\right)=v_{n, p} \in V_{n}\right\} \\
F_{V}:\left(v_{n, p}\right)_{n, p} \longmapsto\left(v_{n, p+1}\right)_{n, p} .
\end{gathered}
$$

The distance on $\underline{X}_{V}$ is defined as: $d(x, y)=\sum_{n \geq 0} 2^{-n} d_{V}\left(x_{-n}, y_{-n}\right)$.

Remark that $\left(\underline{X}_{V}, F_{V}\right)$ is homeomorphic to the usual realization of the natural extension: $\left\{\left(\underline{v}_{p}\right)_{p \in \mathbb{Z}} \in X_{V}^{\mathbb{Z}}: \forall p \in \mathbb{Z} F_{V}\left(\underline{v}_{p-1}\right)=\underline{v}_{p}\right\}$.

The symmetry of the roles of $i$ and $f$ gives rise to a duality between puzzles: just exchange the maps $i$ and $f$ associated to a puzzle $(V, i, f)$. We denote by $\left(V^{*}, i^{*}, f^{*}\right)$ the resulting puzzle. The natural extension of their dynamics $F_{V}$ and $F_{V^{*}}$ are inverse of each other, as the description of the natural extensions given above makes it obvious.

Remark. As it was already the case for subshifts of quasi-finite type [11], $h_{\mathcal{C}}\left(V^{*}\right)$ may be different from $h_{\mathcal{C}}(V)$. It may indeeed occur that $h_{\mathcal{C}}(V)<h_{\text {top }}(V)$ and $h_{\mathcal{C}}\left(V^{*}\right)=h_{\text {top }}\left(V^{*}\right)$ (or the other way around). This allows easy construction of puzzles such that $h_{\text {wloc }}\left(V^{*}\right)$ is different from $h_{\text {wloc }}(V)$. 


\section{Measure-theoretic Structure}

In this section we begin the proof of the structure theorem (Theorem 1). We first introduce the Markov shift which underlies our analysis and then we explain its consequences for entropy-conjugacy. The proof then has three stages: (i) the Markov shift is shown to be measurably conjugate to a part of the natural extension of the puzzle dynamics; (ii) the entropies of the measures living on the excluded part are bounded, yielding claim (1) of the Theorem; (iii) the entropy "at infinity" in the Markov diagram is also controlled, yielding claim (2) of the Theorem. Claim (3), on the periodic points, is proved in the next section.

4.1. The complete Markov diagram. - The key object is the following countable oriented graph.

Definition 4.1. - The complete Markov diagram of a puzzle $V$ is a countable, oriented graph $\mathcal{D}$ defined as follows. Its vertices are the $f$-irreducible vertices of $V$. Its arrows are the following:

$$
v \leadsto w \Longleftrightarrow \exists u \in V i(u)=v \text { and } u \succeq_{f} w .
$$

Notice that because of Lemma 3.1 $u$ in eq. (7) is unique given $v \leadsto w$.

Remark. If $V$ is in fact a subshift over alphabet $V_{1}$, this complete Markov diagram reduces to the one introduced in [6. Under the additional assumption that there are no "accidental" identifications, i.e., $F_{V}^{|v|}([v])=F_{V}^{|w|}([w])$ only if $w=f^{|v|-|w|}(v)$ (assuming $|v| \geq|w|$ ), this further reduces to the Hofbauer diagram [19].

Let $\Sigma_{+}(\mathcal{D})$ be the associated one-sided subshift:

$$
\Sigma_{+}(\mathcal{D})=\left\{\underline{v} \in V^{\mathbb{N}}: v_{0} \leadsto v_{1} \leadsto v_{2} \leadsto \ldots\right\}
$$

together with the left-shift $\sigma\left(\left(v_{n}\right)_{n \in \mathbb{N}}\right)=\left(v_{n+1}\right)_{n \in \mathbb{N}}$.

We build a conjugacy from the Markov shift onto (a part of) the puzzle dynamics.

Proposition 4.2. - Let $\underline{v} \in \Sigma_{+}(\mathcal{D})$ and $n \geq 0$. There exists a unique $w^{(n)} \in V$ such that:

(i) $i^{n}\left(w^{(n)}\right)=v_{0}$;

(ii) for all $k=0, \ldots, n: i^{k}\left(w^{(n)}\right) \succeq_{f} v_{n-k}$.

Moreover, the following property holds:

(iii) $i\left(w^{(n+1)}\right)=w^{(n)}$.

Figure 2 gives a typical example of the construction of $w^{n}$.

Proof: Let $\underline{v} \in \Sigma_{+}(\mathcal{D})$. For each $n \geq 0$, we are going to define $w^{0}, \ldots, w^{n}$ such that:

$$
i^{j}\left(w^{j}\right)=v_{n-j} \text { and } \forall k=0, \ldots, j \quad i^{k}\left(w^{j}\right) \succeq_{f} v_{n-k} .
$$

Observe that $w^{(n)}:=w^{n}$ will then have the required properties (i) and (ii) by eq. (8). (iii) will follow from showing the uniqueness of the solution to (8).

For $j=0$, set $w^{0}=v_{n}$. For $1 \leq j \leq n$, assume that $w^{j-1}$ has been defined satisfying (8). As $v_{n-j} \leadsto v_{n-j+1}$, there exist an integer $l \geq 1$ and $u \in i^{-1}\left(v_{n-j}\right)$ 


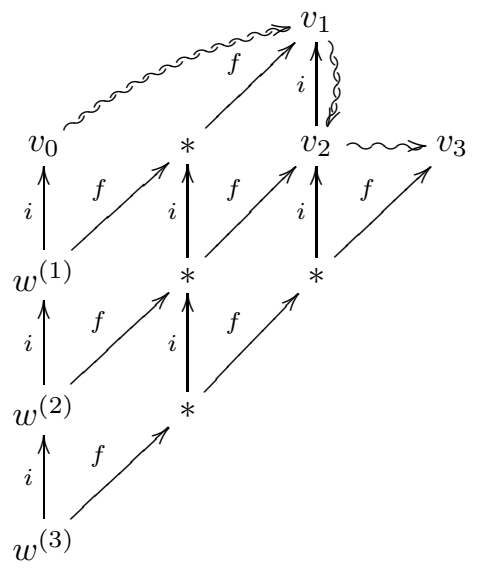

Figure 2. Construction of $w^{(3)}$ from $v_{0} v_{1} v_{2} v_{3}$ as in Proposition 4.2

such that $u \succeq_{f}^{l} v_{n-j+1}$ (where, necessarily, $l=|u|-\left|v_{n-j+1}\right|=\left|v_{n-j}\right|+1-\left|v_{n-j+1}\right|$ ). Hence there exists a $w^{j} \in \mathcal{T}_{i}(u)$ which is the $f^{l}$-preimage of $w^{j-1}$ in $\mathcal{T}_{i}\left(v_{n-j+1}\right)$ (recall that $\left.i^{j-1}\left(w^{j-1}\right)=v_{n-j+1}\right)$.

Let us check (8) for $w^{j}$. Compute

$$
\left|w^{j}\right|=l+\left|w^{j-1}\right|=\left|v_{n-j}\right|+1-\left|v_{n-j+1}\right|+\left(j-1+\left|v_{n-j+1}\right|\right)=\left|v_{n-j}\right|+j .
$$

As $w^{j} \in \mathcal{T}_{i}(u)$ and $i(u)=v_{n-j}$ (i.e., $\left.w^{j} \in \mathcal{T}_{i}\left(v_{n-j}\right)\right)$, this implies the first part of (8) :

$$
i^{j}\left(w^{j}\right)=v_{n-j} .
$$

For the second part, observe that $u \succeq_{f}^{l} v_{n-j+1}, i^{j-1}\left(w^{j}\right)=u, i^{j-1}\left(w^{j-1}\right)=v_{n-j+1}$ and $f^{l}\left(w^{j}\right)=w^{j-1}$. Hence Lemma 3.2 implies that, for $0 \leq k \leq j-1, i^{k}\left(w^{j}\right) \succeq_{f}^{l}$ $i^{k}\left(w^{j-1}\right)$. Using the second part of (8) for $w^{j-1}$ we see that:

$$
\forall 0 \leq k<j \quad i^{k}\left(w^{j}\right) \succeq_{f} i^{k}\left(w^{j-1}\right) \succeq_{f} v_{n-k} .
$$

Thus eq. (8) holds for $w^{j}$ and $k<j$. For $k=j$, this second part is just (9).

This completes the inductive construction of $w^{n}$.

For future reference, observe that $w^{j}$ depends only on $v_{n-j} \ldots v_{n}$ and that the case $k=0$ of the previous equation gives:

$$
w^{j} \succeq_{f} w^{j-1} \succeq_{f} v_{n}
$$

Let us check the uniqueness of problem (8). We prove that for $w^{n}$ satisfying eq. (8), $i^{n-p} w^{n}$ is unique by an induction on $0 \leq p \leq n$. For $p=0$, this is obvious. Assume it for $p-1 \geq 0$. Observe that $i\left(i^{n-p}\left(w^{n}\right)\right)=i^{n-p+1}\left(w^{n}\right)$ and $i^{n-p}\left(w^{n}\right) \succeq_{f} v_{p}$. These two conditions uniquely determine $i^{n-p}\left(w^{n}\right)$ according to Lemma 3.1. Thus $w^{n}$ is indeed unique. 
Thus we have shown the existence of $w^{(n)}$ satisfying properties (i)-(iii) of the statement. We show that $w^{(n)}$ is unique under (i) and (ii). We proceed by induction on $n$. For $n=0$ this is obvious. Assume the uniqueness for $n-1 \geq 0$. Let $w^{\prime}:=i\left(w^{(n)}\right)$. Observe that

$$
\begin{aligned}
& -i^{n-1}\left(w^{\prime}\right)=i^{n}\left(w^{(n)}\right)=v_{0} \\
& \text { - for } 0 \leq k<n, i^{k}\left(w^{\prime}\right)=i^{k+1}\left(w^{(n)}\right) \succeq_{f} v_{n-k-1} .
\end{aligned}
$$

By the induction hypothesis, $w^{\prime}=w^{(n-1)}$. Thus $i\left(w^{(n)}\right)=w^{(n-1)}$ and $w^{(n)} \succeq_{f} v_{n}$. Lemma 3.1 gives the uniqueness of $w^{(n)}$, completing the induction.

Corollary 4.3. - Let $\underline{v} \in \Sigma_{+}(\mathcal{D})$. Then there exists a unique $x \in X_{V}$ such that $x_{\left|v_{0}\right|}=v_{0}$ and for all $j \geq 0, x_{\left|v_{0}\right|+j} \succeq_{f} v_{j}$. Moreover $x_{\left|v_{0}\right|+j}$ depends only on $v_{0} v_{1} \ldots v_{j}$. For future reference we denote this $x \in X_{V}$ by $x(\underline{v})$.

Proof: For each $n \geq 0$, apply the above proposition to the sequence $v_{0} \leadsto \ldots \leadsto v_{n}$ to get $w^{(n)}$. As $i\left(w^{(n+1)}\right)=w^{(n)}$, we define a sequence $x$ in $X_{V}$ by $x_{\left|w^{(n)}\right|}=w^{(n)}$. Moreover, for each $n \geq 0, x_{\left|w^{(n)}\right|-n+j}=i^{n-j}\left(w^{(n)}\right) \succeq_{f} v_{j}$. As $\left|w^{(n)}\right|=\left|v_{0}\right|+n$, this implies that $x_{\left|v_{0}\right|+j} \succeq_{f} v_{j}$.

The uniqueness is proved by applying inductively Lemma 3.1 to $i\left(x_{\left|v_{0}\right|+j+1}\right)=$ $x_{\left|v_{0}\right|+j}$ and $x_{\left|v_{0}\right|+j} \succeq_{f} v_{j}$.

It is obvious that $x_{\left|v_{0}\right|+j}=x_{\left|w^{j}\right|}$ depends only on $v_{0} \ldots v_{j}$.

Let us define $\pi: \Sigma_{+}(\mathcal{D}) \rightarrow X_{V}$ by:

$$
\pi(\underline{v})=F_{V}^{\left|v_{0}\right|}(x(\underline{v}))
$$

with $x(\underline{v})$ defined as in the above Corollary.

Lemma 4.4. - The map $\pi: \Sigma_{+}(\mathcal{D}) \rightarrow X_{V}$ is well-defined, continuous and satisfies: $\pi \circ \sigma=F_{V} \circ \pi$.

Proof: The above Corollary shows that $\pi$ is indeed well-defined and continuous with values in $X_{V}$. We turn to the commutation relation. We must show that

$$
f\left((\pi v)_{n}\right)=(\pi \sigma v)_{n-1}
$$

for all large $n$.

Let $w^{(n+1)}$ be built as in Proposition 4.2 from $v_{0} \ldots v_{n+1}$ using the finite sequence $w^{0}, \ldots, w^{n+1}$ defined in (8). Let $\tilde{w}^{(n)}$ be defined similarly from $v_{1} \ldots v_{n+1}$ using $\tilde{w}^{0}, \ldots, \tilde{w}^{n}$.

Observe that $w^{k}=\tilde{w}^{k}$ for $k \leq n$ as they are both determined by $v_{n+1-k} \ldots v_{n+1}$. According to (10), $w^{(n+1)} \succeq_{f}^{\ell} \tilde{w}^{(n)}$ with $\ell:=\left|w^{(n+1)}\right|-\left|\tilde{w}^{(n)}\right|=\left|v_{0}\right|+1-\left|v_{1}\right| \geq 0$. Hence

$$
f\left(f^{\left|v_{0}\right|}\left(w^{(n+1)}\right)\right)=f^{\left|v_{1}\right|}\left(\tilde{w}^{(n)}\right) .
$$

Now $\pi(v)=F_{V}^{\left|v_{0}\right|}(x)$ with $x_{\left|v_{0}\right|+n+1}=w^{(n+1)}$. Likewise, $\pi(\sigma(v))=F_{V}^{\left|v_{1}\right|}(y)$ with $y_{\left|v_{1}\right|+n}=\tilde{w}^{(n)}$. (11) now follows from the previous equation. 


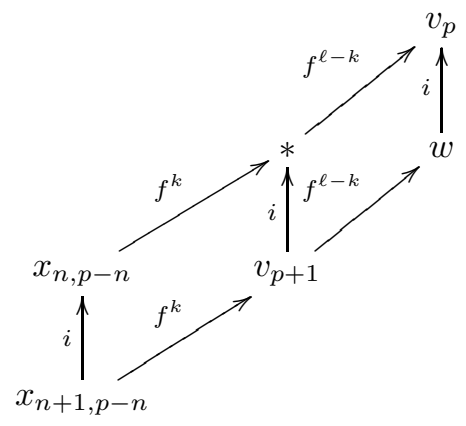

Figure 3. Proof of $v_{p} \leadsto v_{p+1}$ for Proposition 4.6 .

4.2. Partial conjugacy. - We are going to show that $\pi$ gives an isomorphism between a subset of the natural extension $\underline{X}_{V}$ of $X_{V}$ and the whole of $\Sigma(\mathcal{D})$.

Observe that $\pi: \Sigma_{+}(\mathcal{D}) \rightarrow X_{V}$ extends naturally to $\pi: \Sigma(\mathcal{D}) \rightarrow \underline{X}_{V}$ by setting $\pi(v)=x$ with $x_{0 p} x_{1 p} \cdots=\pi\left(v_{p} v_{p+1} \ldots\right)$ because of the commutation in Lemma 4.4.

Definition 4.5. $-x \in \underline{X}_{V}$ is eventually Markovian at time $p$ if there exists $0 \leq N<\infty$ such that:

$$
\forall n \geq N \quad x_{n, p-n} \succeq_{f} x_{N, p-N} .
$$

The eventually Markovian subset $\underline{X}_{V}^{M}$ of $\underline{X}_{V}$ is

$$
\underline{X}_{V}^{M}=\left\{x \in \underline{X}_{V}: x \text { is eventually Markovian at all times }\right\} .
$$

Proposition 4.6. - Define $\iota: \underline{X}_{V}^{M} \rightarrow \Sigma(\mathcal{D})$ by $\iota(x)=v$ if, for all $p \in \mathbb{Z}, v_{p}$ is the unique irreducible vertex such that for all sufficiently large $n$ :

$$
x_{n, p-n} \succeq_{f} v_{p} .
$$

Then $\iota:\left(\underline{X}_{V}^{M}, F_{V}\right) \rightarrow(\Sigma(\mathcal{D}), \sigma)$ is well-defined and gives an isomorphism whose inverse is $\pi$.

Proof: Let us first check that $\iota$ is well-defined with $\iota\left(\underline{X}_{V}^{M}\right) \subset \Sigma(\mathcal{D})$. Let $x \in \underline{X}_{V}^{M}$. $\underline{X}_{V}^{M}$ is precisely defined so that $v=\iota(x)$ is a well-defined element of $\mathcal{D}^{\mathbb{Z}}$. (12) gives uniqueness at once.

Let us show that $v_{p} \leadsto v_{p+1}$ for an arbitrary $p \in \mathbb{Z}$. For $n$ large enough,

$$
\text { (i) } x_{n, p-n} \succeq_{f}^{\ell} v_{p} \text { and (ii) } x_{n+1, p+1-n-1}=x_{n+1, p-n} \succeq_{f}^{k} v_{p+1}
$$

where $\ell=\left|x_{n, p-n}\right|-\left|v_{p}\right|$ and $k=\left|x_{n+1, p-n}\right|-\left|v_{p+1}\right|$.

Let $u=f^{\ell}\left(x_{n+1, p-n}\right)$ (note that $\left|x_{n+1, p-n}\right|=n+1>n>\ell$ ). We have $i(u)=$ $f^{\ell}\left(i\left(x_{n+1, p-n}\right)\right)=f^{\ell}\left(x_{n, p-n}\right)=v_{p}$. Hence it is enough to see that $u \succeq_{f} v_{p+1}$. Given (13)ii), this will follow from $\ell \leq k$. If $\ell>k, x_{n+1, p-n} \succeq_{f}^{\ell} u$ (a consequence of $x_{n, p-n} \succeq_{f} v_{p}$ according to Lemma 3.2 and $x_{n+1, p-n} \succeq_{f}^{k} v_{p+1}$ would imply: $v_{p+1} \succeq_{f}^{\ell-k}$ $u$, contradicting the irreducibility of $v_{p+1}$. Thus $\iota(x) \in \Sigma(\mathcal{D})$. 
Let us prove that $\iota \circ \pi=\operatorname{Id}_{\Sigma(\mathcal{D})}$. Let $v \in \Sigma(\mathcal{D})$ and $x=\pi(v) \in \underline{X}_{V}$. Let us check that $x$ belongs to $\underline{X}_{V}^{M}$. We have, for $p \in \mathbb{Z}$ and $n \geq 1$,

$$
x_{n, p-n}=\left(\pi\left(v_{p-n} v_{p-n+1} \ldots\right)\right)_{n}=f^{\left|v_{p-n}\right|}\left(y_{\left|v_{p-n}\right|+n}\right)
$$

where $y_{\left|v_{p-n}\right|+n} \succeq_{f}^{k} v_{p}$ for $k=\left|v_{p-n}\right|+n-\left|v_{p}\right|$ by Corollary 4.3. For $n \geq\left|v_{p}\right|$, $k \geq\left|v_{p-n}\right|$ and

$$
x_{n, p-n}=f^{\left|v_{p-n}\right|}\left(y_{\left|v_{p-n}\right|+n}\right) \succeq_{f} v_{p} .
$$

Thus $x$ is eventually Markov at any time $p . x \in \underline{X}_{V}^{M}$ as claimed. Observe that eq. (14) also implies that $\iota(x)=v$, i.e., $\iota \circ \pi=\operatorname{Id}_{\Sigma(\mathcal{D})}$ as claimed.

It remains to show that $\iota: \underline{X}_{V}^{M} \rightarrow \Sigma(\mathcal{D})$ is one-to-one. Let $x, y \in \underline{X}_{V}^{M}$ with $\iota(x)=\iota(y)=: v$. Let $p \in \mathbb{Z}$. As $v_{p} \leadsto v_{p+1}$, there is a unique $u_{p}^{1}$ such that $i\left(u_{p}^{1}\right)=v_{p}$ and $u_{p}^{1} \succeq_{f}^{k} v_{p+1}$ for $k=\left|v_{p}\right|+1-\left|v_{p+1}\right|$ by Lemma 3.1.

For $n$ large enough, we have $x_{n, p-n}, y_{n, p-n} \succeq_{f}^{\ell} v_{p}$ for $\ell=n-\left|v_{p}\right|$ and $x_{n+1, p-n}, y_{n+1, p-n} \succeq_{f}$ $v_{p+1}$. Then $x_{n+1, p-n} \succeq_{f}^{\ell} x_{n+1-\ell, p-n+\ell}=: w$ and $w$ must satisfy $i(w)=v_{p}$ and $w \succeq_{f} v_{p+1}$ (observe that $|w|=n+1-\ell=\left|v_{p}\right|+1 \geq\left|v_{p+1}\right|$ ). By Lemma 3.1. $w=x_{n+1, p-n}$. Thus

$$
x_{n+1, p-n}, y_{n+1, p-n} \succeq_{f} u_{p}^{1}=x_{\left|v_{p}\right|+1, p-\left|v_{p}\right|}=y_{\left|v_{p}\right|+1, p-\left|v_{p}\right|} .
$$

We want to repeat this analysis with $u^{1}$ replacing $v_{p}$. First we check that $u_{p}^{1} \leadsto u_{p+1}^{1}$, i.e., that there is some $w$ such that $i(w)=u_{p}^{1}$ and $w \succeq_{f} u_{p+1}^{1}$ (but some $u_{p}^{1}$ might be reducible). Indeed, $f^{k}: \mathcal{T}_{i}\left(u_{p}^{1}\right) \rightarrow \mathcal{T}_{i}\left(v_{p+1}\right)$ is an isomorphism so that there exists $w \in \mathcal{T}_{i}\left(u_{p}^{1}\right)$ with $f^{k}(w)=u_{p+1}^{1}$. Lemma 3.2 gives then that $w \succeq_{f} u_{p+1}^{1}$. But this says that $u_{p}^{1} \leadsto u_{p+1}^{1}$, as claimed.

We proceed inductively, assuming that some sequence $\left(u_{p}^{j}\right)_{p \in \mathbb{Z}}$ has been obtained such that $u_{p}^{j} \leadsto u_{p+1}^{j}$ and

$$
x_{n+j, p-n} \succeq_{f} u_{p}^{j}=x_{\left|u_{p}^{j}\right|+1, p-\left|v_{p}\right|}=y_{\left|u_{p}^{j}\right|+1, p-\left|v_{p}\right|}
$$

We define $u_{p}^{j+1}$ as the unique piece such that $i\left(u_{p}^{j+1}\right)=u_{p}^{j}$ and $u_{p}^{j+1} \succeq_{f} u_{p+1}^{j}$. The same reasoning as above yields (15) with $j$ replaced by $j+1$.

As $\left|u_{p}^{j}\right|=\left|v_{p}\right|+j \rightarrow \infty$, we obtain $x=y$.

Corollary 4.7. - The induced maps on the invariant probability measures $\pi: \operatorname{Prob}(\sigma, \Sigma(\mathcal{D})) \rightarrow$ $\operatorname{Prob}\left(F_{V}, \underline{X}_{V}\right)$ and $\pi: \operatorname{Prob}\left(\sigma, \Sigma_{+}(\mathcal{D})\right) \rightarrow \operatorname{Prob}\left(F_{V}, X_{V}\right)$ are one-to-one and preserve ergodicity and entropy.

Proof: That $\pi: \Sigma(\mathcal{D}) \rightarrow \underline{X}_{V}$ is a partial isomorphism trivially implies the stated properties of $\pi: \operatorname{Prob}(\sigma, \Sigma(\mathcal{D})) \rightarrow \operatorname{Prob}\left(F_{V}, \underline{X}_{V}\right)$. To finish, recall that the natural extension construction preserves ergodicity and entropy. 


\subsection{Entropy of the non-Markov part. -}

Proposition 4.8. - If $\mu$ is an invariant and probability measure with $\mu\left(\underline{X}_{V} \backslash \underline{X}_{V}^{M}\right)=$ 0 , then

$$
h\left(F_{V}, \mu\right) \leq h_{\mathcal{C}}(V) .
$$

To analyze the non-Markov part, the first step is the following:

Lemma 4.9. - Up to a set of zero measure with respect to any invariant probability measure, each $x \in \underline{X}_{V} \backslash \underline{X}_{V}^{M}$ satisfies: for all $p \in \mathbb{Z}$ there exist arbitrarily large integers $n$ such that $x_{n, p-n}$ is an $f$-irreducible vertex.

Proof of the Lemma: By definition $x \in \underline{X}_{V} \backslash \underline{X}_{V}^{M}$ iff there exists $p \in \mathbb{Z}$ as in the statement of the Lemma. Let $X(p)$ be the set of such $x$. The lemma is clearly equivalent to the fact that, for any invariant probability $\mu$,

$$
\mu\left(\bigcup_{p \in \mathbb{Z}} X(p)\right)=\mu\left(\bigcap_{p \in \mathbb{Z}} X(p)\right) .
$$

It is enough to prove this for ergodic $\mu$ 's such that the union has positive and hence full measure. If we prove that $X(p+1) \subset X(p)$, it will follow that $\mu\left(\bigcup_{p \in \mathbb{Z}} X(p)\right)=$ $\lim _{p \rightarrow-\infty} \mu(X(p))$ which is equal to $\mu(X(p))$ for any $p \in \mathbb{Z}$ by invariance of $\mu$, proving (16). But observe that by Lemma 3.2

$$
\left.x_{n, p-n} \succeq_{f} x_{N, p-N} \Longrightarrow x_{n+1, p+1-(n+1)} \succeq_{f} x_{N+1, p+1-(N+1)}\right)
$$

so that $x \notin X(p) \Longrightarrow x \notin X(p+1)$, which concludes the proof.

Recall that the entropy of an invariant and ergodic probability measure $\mu$ can be computed as 23:

$$
\begin{aligned}
& h\left(F_{V}, \mu\right)=\lim _{\epsilon \rightarrow 0} h\left(F_{V}, \mu, \epsilon\right) \text { with } \\
& \qquad h\left(F_{V}, \mu, \epsilon\right)=\limsup _{n \rightarrow \infty} \frac{1}{n} \log \min \left\{\# S: \mu\left(\bigcup_{x \in S} B(x, \epsilon, n)\right)>\mu_{0}\right\}
\end{aligned}
$$

where $0<\mu_{0}<1$ is arbitrary.

Proof of the Proposition: Let $\mu$ be an invariant probability measure carried by $\underline{X}_{V} \backslash \underline{X}_{V}^{M}$. We may and do assume that $\mu$ is ergodic. Let $\alpha>0$ be some small number. There exists $r>0$ (depending on $\mu$ ) such that $h\left(F_{V}, \mu\right) \leq h\left(F_{V}, \mu, r\right)+\alpha$. Fix $L_{1}<\infty$ and $r_{1}>0$ such that for $x, y \in \underline{X}_{V}, d\left(x_{2 L_{1},-L_{1}}, y_{2 L_{1},-L_{1}}\right)<r_{1} \Longrightarrow d(x, y)<r$ (for any distance on $X_{V}$ and $\underline{X}_{V}$ compatible with the topologies). Let $L_{2}$ be such that $r\left(r_{1}, n, \mathcal{C}_{n}\right) \leq e^{\left(h_{\mathcal{C}}(V)+\alpha\right) n}$ for all $n \geq L_{2}$ and fix, for each such $n$, some $\left(r_{1}, n\right)$-cover ${ }^{(4)}$ $C_{n}$ of $\mathcal{C}_{n}$ with this minimum cardinality. For each $v \in C_{n}$, we pick some $x \in \underline{X}_{V}$ such that $x_{n, 0}=v$ and let $X_{n}:=\left\{x^{v} \in X_{V}: v \in C_{n}\right\}$.

${ }^{(4)}$ Recall the definition of the entropy of sequences. 
Let $L>>L_{1} \log K / 2 \alpha+L_{2}$ where $K$ is the minimum cardinality of an $r$-dense subset of $\underline{X}_{V}$. It follows from Lemma 4.9 that there exists a measurable function $n: \underline{X}_{V} \rightarrow \mathbb{N}$ such that, for $\mu$-a.e. $\underline{x} \in \underline{X}_{V}$ :

$-n(\underline{x}) \geq L$

- $x_{n,-n}$ is $f$-irreducible for $n=n(\underline{x})$.

Hence (see [11, p. 394]) a $\mu$-typical $x$ satisfies the following. For all large $n$, there exist disjoint integer intervals $\left[a_{i}, b_{i}\right) \subset[0, n), i=1, \ldots, s$, such that

1. $\sum_{i=1}^{s} b_{i}-a_{i} \geq(1-\alpha) n$;

2. $b_{i}-a_{i} \geq L$ for all $i=1, \ldots, s$;

3. $x_{b_{i}-a_{i}+1, a_{i}} \nsucceq_{f} x_{b_{i}-a_{i}, a_{i}+1}$ : in particular, $x_{b_{i}-a_{i}+1, a_{i}}$ is $f$-irreducible. Thus $F_{V}^{a_{i}+L_{1}}(x) \in B\left(y, r, b_{i}-a_{i}-2 L_{1}\right)$ for some $y \in X_{b_{i}-a_{i}+1}$.

It follows (see, e.g., the same reference) that $h\left(F_{V}, \mu\right) \leq h_{\mathcal{C}}(V)+3 \alpha+\alpha|\log \alpha| \leq H$. As $\alpha>0$ is arbitrarily small, this concludes the proof.

\subsection{Entropy at infinity in the diagram. -}

Proposition 4.10. - Let $H>h_{C}(V)+h_{\mathrm{wloc}}(V)$. Then there exists a finite subset $\mathcal{D}_{0} \subset \mathcal{D}$ such that:

$h\left(\mathcal{D} \backslash \mathcal{D}_{0}, \mathcal{D}\right):=\inf _{\mu_{0}>0} \sup \left\{h(\sigma, \mu): \mu \in \operatorname{Prob}_{\operatorname{erg}}(\Sigma(\mathcal{D}))\right.$ and $\left.\mu\left(\bigcup_{D \in \mathcal{D}_{0}}[D]\right)<\mu_{0}\right\} \leq H$

where $\operatorname{Prob}_{\operatorname{erg}}(\Sigma(\mathcal{D}))$ is the set of shift-invariant and ergodic probability measures on $\Sigma(\mathcal{D})$.

Proof: It is enough to find $\mathcal{D}_{0}$ and $\mu_{0}>0$ such that if $\mu \in \operatorname{Prob}_{\text {erg }}(\Sigma(\mathcal{D}))$ satisfies:

$$
\mu\left(\bigcup_{D \in \mathcal{D}_{0}}[D]\right)<\mu_{0}
$$

then $h(\sigma, \mu) \leq H$.

Let $\alpha>0$ be so small that $h_{\mathcal{C}}(V)+h_{\text {wloc }}(V)+4 \alpha+\alpha|\log \alpha| \leq H$. Let $r>0$ be such that, for all invariant and ergodic probability measures $\mu$ with $h\left(F_{V}, \mu\right)>h_{\mathcal{C}}(V)$ :

$$
h\left(F_{v}, \mu\right)-h\left(F_{V}, \mu, r\right) \leq h_{\text {wloc }}\left(F_{V}\right)+\alpha
$$

(the point here is that $r$ and therefore $L_{1}$ and $r_{1}$ are now fixed, especially they are independent from $\mu$-compare with Proposition 4.6). Fix $L_{1}<\infty$ and $r_{1}>0$ such that, for all $x, y \in \underline{X}_{V}, d\left(x_{2 L_{1},-L_{1}}, y_{2 L_{1},-L_{1}}\right)<r_{1} \Longrightarrow d(x, y)<r$. We increase $L_{1}$ if necessary so that $L_{1}>r_{1}^{-1}$. Recall that $\mathcal{C}=\left(C_{n}\right)_{n \geq 1}$ with $C_{n}$ the set of irreducible vertices of order $n$. Let $L_{2}$ such that $r\left(r_{1}, n, \mathcal{C}_{n}\right) \leq e^{\left(h_{\mathrm{top}}(\mathcal{C})+\alpha\right) n}$ for all $n \geq L_{2}$. Let $K$ be the cardinality of a finite $r$-dense subset of $\underline{X}_{V}$ and let $L>\alpha^{-1} L_{1} \log K+L_{2}$.

Finally let

$$
\mathcal{D}_{0}=\{v \in \mathcal{D}:|v| \leq L\}
$$

and let $\mu_{0}>0$ be a very small number to be specified later.

Let $\mu$ be an ergodic invariant probability measure on $\Sigma(\mathcal{D})$ satisfying (17). We bound $h(\sigma, \mu)$. First observe that by Corollary 4.7, $h(\sigma, \mu)=h\left(F_{V}, \pi_{*} \mu\right)$. Let $x \in \underline{X}_{V}$ 
be a $\pi_{*} \mu$-typical point. Thus $x=\pi(v)$ with $v$ a path on $\mathcal{D}$ spending a fraction of its time less than $\mu_{0}$ in $\mathcal{D}_{0}$.

This implies that there exist disjoint integer intervals $\left[a_{1}, b_{1}\right), \cdots \subset[0, n)$ with $v_{b_{i}} \in \mathcal{D} \backslash \mathcal{D}_{0}$ such that $\sum_{i} b_{i}-a_{i} \geq\left(1-\mu_{0}\right) n$ and $x_{\left|v_{b_{i}}\right|, b_{i}-\left|v_{b_{i}}\right|}=v_{b_{i}}$. The latter implies: $d\left(x_{a_{i}+k}, f^{k}\left(v_{b_{i}}\right)\right)<r_{1}$ for all $k \in\left[0, b_{i}-a_{i}-L_{1}\right)$. Note that the $b_{i}-a_{i}$ are large (larger than $L$ ). By definition of $\mathcal{D}$, the $v_{b_{i}}$ s are $f$-irreducible. It follows as in the proof of Proposition 4.8 that

$h\left(F_{V}, \pi_{*} \mu, r\right) \leq h_{\mathcal{C}}(V)+\alpha+\frac{1}{L}+\frac{1}{L}\left|\log \frac{1}{L}\right|+\left(\mu_{0}+2 L_{1} / L\right) \log K \leq h_{\mathcal{C}}(V)+3 \alpha+\alpha|\log \alpha|$ if $\mu_{0}=\mu_{0}(V, r, \alpha)$ is small enough. If $h\left(F_{V}, \pi_{*} \mu\right) \leq h_{\mathcal{C}}(V) \leq H$, there is nothing to show. Otherwise,

$$
h\left(F_{V}, \pi_{*} \mu\right) \leq h_{\mathcal{C}}(V)+h_{\mathrm{wloc}}\left(F_{V}, r\right)+4 \alpha+\alpha|\log \alpha| \leq H
$$

as claimed.

4.5. Conclusion of the Analysis of Large Entropy Measures. - We collect all the partial results and check that they imply the first two claims of Theorem 1

First, let $V$ be a $*$-QFT puzzle. Propositions 4.6 and 4.8 immediately imply that $\underline{X}_{V}$ is entropy-conjugate with constant $h_{\mathcal{C}}(V)$ to the Markov shift, $\Sigma(\mathcal{D})$, proving claim (1) of the Theorem.

For claim (2), we assume that $V$ is QFT $: h_{\text {top }}\left(X_{V}\right)>H_{*}:=h_{\mathcal{C}}(V)+h_{\text {wloc }}\left(X_{V}\right)$. Proposition 4.10 implies that $h_{\infty}(\mathcal{D}) \leq H_{*}$.

Take $H$ strictly between $H_{*}$ and $h_{\text {top }}(V): \mathcal{D}$ contains only finitely many irreducible Markov subshifts $S$ with entropy $h(S) \geq H$. This implies that $h(S)>H_{*} \geq h_{\infty}(\mathcal{D}) \geq$ $h_{\infty}(S)$. Hence, by the result of Gurevič and Zargaryan [18, quoted in Proposition 6.1 below these irreducible subshifts are SPR. This proves claim (2) of Theorem1.

\section{Periodic Structure}

In this section we prove Claim (3) of Theorem 1 which relates most periodic orbits in the Markov shift with most periodic orbits in some fine scale approximation $i_{N}\left(X_{V}\right)$ of the puzzle dynamics $X_{V}$. It is here that we need determinacy, exactly once, to prove eq. (18).

5.1. Partition of the periodic points. - The proof will use two integer parameters $N, L \geq 1$ depending on $\epsilon>0$. We shall denote $i_{N} \circ \pi: \Sigma(\mathcal{D}) \rightarrow i_{N}\left(\underline{X}_{V}\right)$ by $\pi_{N}$. The $n$-fixed points $\xi=\sigma^{n}(\xi)$ of $i_{N}\left(X_{V}\right)$ satisfy exactly one of the following properties:

(P1) there exist $v \in \pi_{N}^{-1}(\xi) \in \Sigma(\mathcal{D})$ such that $I_{N}(v):=\left\{p \geq 0:\left|v_{p}\right|<N\right\}$ is infinite.

(P2) $\pi_{N}^{-1}(\xi) \neq \emptyset$ but for all $v$ in this set, $I_{N}(v)$ is finite.

(P3) $\pi_{N}^{-1}(\xi)=\emptyset$.

Denote by $\widetilde{\operatorname{Fix}_{i}}(n), i=1,2,3$, the corresponding sets of periodic points of $i_{N}\left(X_{V}\right)$ (these sets do not depend on $L$, which will define a splitting of $\widetilde{\operatorname{Fix}_{2}}(n)$ below). 
On the other hand, we consider on the Markov shift only the periodic points defined by low loops:

$$
\widehat{\operatorname{Fix}}_{1}(n):=\left\{v \in \Sigma(\mathcal{D}): \sigma^{n} v=v \text { and }\left\{v_{0}, \ldots, v_{n-1}\right\} \cap \mathcal{D}_{N} \neq \emptyset\right\} .
$$

We shall say nothing about the others.

5.2. Low loops and periodic points of $i_{N}\left(X_{V}\right)$. - Let $\epsilon>0, N_{0}$ and $\mathcal{D}_{0}$ be given as in the statement of the Theorem. Fix $N \geq N_{0}$ so that $\mathcal{D}_{N} \supset \mathcal{D}_{0}$ and $h\left(\mathcal{D} \backslash \mathcal{D}_{N}\right) \leq h_{\mathcal{C}}(V)+h_{\text {wloc }}(V)+\epsilon / 2$ (which is possible by Proposition 4.10 as $h(\mathcal{D} \backslash$ $\left.\left.\mathcal{D}_{N}\right) \leq h\left(\mathcal{D} \backslash \mathcal{D}_{N}, \mathcal{D}\right)\right)$.

We first claim that for all $n \geq 1$ :

$$
\# \widetilde{\mathrm{Fix}}_{1}(n)=\# \widehat{\mathrm{Fix}}_{1}(n)
$$

We need the following consequence of determinacy:

Lemma 5.1. - Let $V$ be a determined puzzle and $N \geq 1$. Let $v, v^{\prime} \in \Sigma(\mathcal{D})$. If $x=\pi(v)$ and $x^{\prime}=\pi\left(v^{\prime}\right)$ satisfy $i_{1}(x)=i_{1}\left(x^{\prime}\right)$, then:

$$
v_{0}=v_{0}^{\prime} \Longrightarrow \forall n \geq 0 x_{n,-n}=x_{n,-n}^{\prime}
$$

ProOF: For $n=n_{0}:=\left|v_{0}\right|$, the right hand side of (19) follows from $v_{0}=x_{n_{0},-n_{0}}=$ $x_{n_{0},-n_{0}}^{\prime}$, which holds by (12). This implies (19) for $n \leq n_{0}$. Assuming it for some $n \geq n_{0}$, (12) again implies $x_{n+1,-n-1}, x_{n+1,-n-1}^{\prime} \succeq_{f} x_{n,-n}=x_{n,-n}^{\prime}$. Together with the determinacy and $i_{1}\left(x_{n+1,-n-1}\right)=i_{1}\left(x_{n+1,-n-1}^{\prime}\right)$, this completes the induction and the proof of the lemma.

We deduce (18) from this Lemma. Let

$$
\Sigma(N):=\left\{v \in \Sigma(\mathcal{D}): \exists p \rightarrow \infty\left|v_{p}\right|<N\right\} .
$$

By Lemma 5.1, $\pi_{N} \mid \Sigma(N)$ is one-to-one. $\widetilde{\mathrm{Fix}}_{1}(n)$ is by definition the set of fixed points $\xi$ of $\sigma^{n}$ in $\pi_{N}(\Sigma(N))$. By the injectivity of $\pi_{N} \mid \Sigma(N)$ and the $\sigma$-invariance of $\Sigma(N)$, such $\xi$ are the $\pi_{N}$ images of the fixed points of $\sigma^{n}$ in $\pi_{N}(\Sigma(N))$. This proves the claim (18).

5.3. Remaining loops and periodic points. - To conclude we check that the remaining factors are holomorphic.

Lemma 5.2. - For every $\epsilon>0$, there exists $N_{0}(\epsilon)<\infty$ such that, for all $N \geq$ $N_{0}(\epsilon)$, for all $n \geq 1$ :

$$
\begin{aligned}
& \# \widetilde{\operatorname{Fix}}_{2}(n) \leq C(N, \epsilon) e^{\left(h_{\mathcal{C}}(V)+\epsilon\right) n} \\
& \# \widetilde{\operatorname{Fix}}_{3}(n) \leq C(N, \epsilon) e^{\left(h_{\mathcal{C}}(V)+\epsilon\right) n}
\end{aligned}
$$

Remark. Of course, $N$ being large, $h\left(\Sigma\left(\mathcal{D} \backslash \mathcal{D}_{N}\right)\right)<H:=h_{\mathcal{C}}(V)+h_{\text {wloc }}(V)+\epsilon<$ $h_{\text {top }}(V)$ but this is unsufficient to prove (20) as Gurevič entropy only controls the number of loops based at a fixed vertex. Indeed, in some examples, $\mathcal{D} \backslash \mathcal{D}_{N}$ contains infinitely many loops of each length.

This Lemma will be enough to conclude the proof of Theorem 3 . 
5.4. Paths above $N$. - To prove (20) we bound the number of the $n$-periodic projections to $i_{N}\left(X_{V}\right)$ of (not necessarily periodic) paths on $\mathcal{D} \backslash \mathcal{D}_{N}$.

The proof is similar to that of Proposition 4.10. For $n \geq N$, let

$$
C(n):=\left\{\left(i_{N}(v), i_{N}(f(v)), \ldots, i_{N}\left(f^{n-N}(v)\right)\right) \in V_{\leq N}^{n-N}: v \in \mathcal{C}_{n}\right\} .
$$

By (12), for all $w \in \Sigma(\mathcal{D})$, writing $y:=\pi(w) \in \underline{X}_{V}$,

(23) $w_{0}$ irreducible with $\ell:=\left|w_{0}\right| \geq N \Longrightarrow\left(y_{N,-\ell+1}, y_{N,-\ell+2}, \ldots, y_{N,-N}\right) \in C(\ell)$

so that $\{C(n): n \geq 1\}$ controls the projections of high paths. By definition,

$$
\# C(n) \leq C(N, \epsilon) e^{\left(h_{\mathcal{C}}(V)+\epsilon\right) n},
$$

hence this control should give an entropy bound. Let us see the details (note that the bound proved below is only $h_{\mathcal{C}}(V)+h_{\text {wloc }}(V)$ ). We fix $L=L(N, \epsilon)$, a large integer.

Let $n \geq 1$ and $\xi \in i_{N}\left(\underline{X}_{V}\right)$ with $\sigma^{n}(\xi)=\xi$ satisfying (P2): $\xi=\pi_{N}(v)$ for some $v \in \Sigma(\mathcal{D})$ satisfying: $\left|v_{p}\right| \geq N$ for all $p \geq p_{0}$. By periodicity of $\xi$, we can assume $p_{0}=0$ by shifting $v$ a multiple of the period. We shift again $\xi$ to ensure $I_{L}(v)=\emptyset$ if $I_{L}(v)$ is finite, $\left|v_{0}\right| \leq L$ otherwise (this might produce an irrelevant factor $n$ in the estimates). Let $x=\pi_{N}(v) \in \underline{X}_{V}$.

Define inductively the integers $r \geq 1, n>b_{1}>\cdots>b_{r} \geq 0$ as follows. $b_{1}:=n-1$. If $b_{i}-\left|v_{b_{i}}\right| \geq 0$ and $\left|v_{b_{i}}\right|>L$, then set $b_{i+1}:=b_{i}-\left|v_{b_{i}}\right| \geq 0$, otherwise let $r:=i$. Finally set $\ell_{i}:=\left|v_{b_{i}}\right|$ for all $i=1,2, \ldots, r$.

Now, by (23), $x_{N, b_{i}-\ell_{i}+k}=i_{N} \circ f^{k}\left(v_{b_{i}}\right)$ for $0 \leq k<\ell_{i}-N$. Thus, there exists $x(i) \in C\left(\ell_{i}\right)$, such that $\xi_{b_{i}-\ell_{i}+k}=(x(i))_{k}$ for $0 \leq k \leq \ell_{i}-N$.

Notice that $\ell_{i} \geq L$ for $i=1, \ldots, r-1$. Hence, given $n \geq 1$ and $b_{r}$, when $v$ ranges over $\Sigma\left(\mathcal{D} \backslash \mathcal{D}_{N}\right)$, the number of choices for the integers $b_{1}, \ldots, b_{r-1}$ is at most $e^{\epsilon\left(n-b_{r}\right)}$ as $L$ is large. On the other hand, eq. (24) yields, for any $\ell \geq 0$,

$\#\left\{\xi_{-\ell+1} \ldots \xi_{0}: \xi=\pi_{N}(v)\right.$ with $v \in \Sigma(\mathcal{D})$ and $\left.\left|v_{0}\right|=\ell\right\} \leq C(N, \epsilon) e^{\left(h_{\mathcal{C}}(V)+\epsilon\right) \ell} \times\left(\# V_{\leq N}\right)^{N}$

This implies:

$$
\begin{aligned}
& \#\left\{\xi_{b_{r}} \ldots \xi_{n-1}: \xi \in \widetilde{\operatorname{Fix}_{2}}(n) \text { with given } b_{r} \text { and } I_{N}(v)=\emptyset\right\} \leq \\
& \begin{aligned}
\left(\# V_{\leq N}\right)^{N\left(n-b_{r}\right) / L} \cdot e^{\epsilon\left(n-b_{r}\right)} \cdot C(N, \epsilon)^{\left(n-b_{r}\right) / L} \exp \left(h_{\mathcal{C}}(V)+\epsilon\right)\left(n-b_{r}\right) \\
\leq C e^{\left(h_{C}(V)+3 \epsilon\right)\left(n-b_{r}\right)}
\end{aligned}
\end{aligned}
$$

using that $L=L(N, \epsilon)$ is large. Note that there are at most $n$ possibilities for $b_{r}$. It remains to count the possibilities for $\xi_{0} \ldots \xi_{b_{r}-1}$. There are two cases.

First case: $\left|v_{b_{r}}\right| \leq L .{\widetilde{\operatorname{Fix}_{2}}}_{2}^{(1)}(n)$ be the corresponding subset of $\widetilde{\operatorname{Fix}_{2}}(n)$. Recall that in this case $I_{L}(v)$ is infinite and $\left|v_{0}\right| \leq L$. Hence $v_{0} \ldots v_{b_{r}}$ is a path on $\mathcal{D} \backslash \mathcal{D}_{N}$ which starts and ends in the finite subgraph $\mathcal{D}_{L} \backslash \mathcal{D}_{N}$. The number of such paths with given $b_{r}$ is bounded using the Gurevič entropy by:

$$
\left(\# \mathcal{D}_{L} \backslash \mathcal{D}_{N}\right)^{2} e^{\left(h\left(\mathcal{D} \backslash \mathcal{D}_{N}\right)+\epsilon / 2\right) b_{r}} \leq\left(\# \mathcal{D}_{L} \backslash \mathcal{D}_{N}\right)^{2} e^{\left(h(V)+h_{\text {wloc }}(V)+\epsilon\right) b_{r}} .
$$


Combining with eq. (25) and summing over $1 \leq r \leq n / L+1$ and $0 \leq b_{r} \leq n$ we obtain:

$$
\# \widetilde{\operatorname{Fix}}_{2}^{(1)}(n) \leq C^{\prime}(L, N, \epsilon) n^{2} e^{\left(h(V)+h_{\text {wloc }}(V)+3 \epsilon\right) n} .
$$

Second case: $\left|v_{b_{r}}\right|>L$ and $b_{r}-\left|v_{b_{r}}\right|=:-\delta<0$. Let $\widetilde{\operatorname{Fix}}_{2}^{(2)}(n)$ be the corresponding subset of $\widetilde{\operatorname{Fix}_{2}}(n)$. We shift $\xi$ by $\delta$ (and add $\delta$ to each $b_{i}$ ) so $b_{r}-\left|v_{b_{r}}\right|=0$ (doing this we lose the property $\left|v_{0}\right| \leq L$ if $I_{L}(v)$ is infinite). Of course, $b_{1}, \ldots, b_{s} \geq n$ for some (maximum) $s \geq 1$. We forget about $b_{1}, \ldots, b_{s-1}$ and we trim $v_{b_{s}}$ in the following way. We replace $b_{s}, v_{b_{s}}$ and $\ell_{s}$ by $n-1, v_{*}:=i^{b_{s}-n+1}\left(v_{b_{s}}\right)$ and $\ell_{*}:=\ell_{s}-\left(b_{s}-n+1\right) \geq 1$. We have now that $x_{-N, b_{s}-\ell_{s}+i}=i_{N}\left(f^{i} v_{*}\right)$ for $0 \leq i<\ell_{*}-N$. We may now apply (25) with $b_{r}=0$. This concludes the proof of (20).

5.5. Unliftable periodic orbits. - Let $\xi \in \widetilde{\operatorname{Fix}_{3}}(n)$. By definition, for any $x \in$ $\underline{X}_{V}$ projecting to $\xi$, there exist $p \in \mathbb{Z}$ and arbitrarily large integers $k$ such that, $x_{k, p-k}$ is $f$-irreducible. Take such an integer $k \geq N+n$ and observe that $v_{*}:=x_{N+n, p-k}$ is $f$-irreducible by Lemma 3.2. Hence, setting $q:=p-k-\left|v_{*}\right|$,

$$
\xi_{q+i}=i_{N}\left(f^{i}\left(v_{*}\right)\right) \text { for all } 0 \leq i<n .
$$

This implies that

$$
\# \widetilde{\operatorname{Fix}_{3}}(n) \leq C(N, \epsilon) e^{\left(h_{\mathcal{C}}(V)+\epsilon\right) n}
$$

proving eq. (21) and concluding the proof of Theorem 3

\section{Semi-local zeta functions of SPR Markov shifts}

We give a proof of Theorem 4 about the meromorphy of the semi-local zeta functions of SPR Markov shifts, after recalling the relation between the entropy at infinity and the SPR property.

6.1. SPR property and entropy at infinity. - A combinatorial quantity related to our $h_{\infty}(G)$ first appeared in a work of Gurevič-Zargaryan [18 to give a sufficient condition for being SPR, which was then shown to be necessary (see [17, Theorem 3.8]). The explicit relation (26) below is due to Ruette [34.

\section{Proposition 6.1 (Gurevič-Zargaryan, Gurevič-Savchenko, Ruette)}

Let $G$ be a countable, oriented, irreducible graph with $h(G)<\infty$. The graph $G$ is SPR iff $h_{\infty}(G)<h(G)$ where the entropy at infinity $h_{\infty}(G)$ has been defined in 9 and can be computed as:

$$
\begin{aligned}
h_{\infty}(G)=\inf _{F \subset \subset} \max _{u, v \in F} \limsup _{n \rightarrow \infty} \frac{1}{n} \log \#\left\{\left(x_{0}, \ldots, x_{n}\right) \in\{u\} \times G^{n-1} \times\{v\}:\right. \\
\left.\forall i=0, \ldots, n x_{i} \rightarrow x_{i+1} \text { on } G \text { and }\left\{x_{1}, x_{2}, \ldots, x_{n-1}\right\} \cap F=\emptyset\right\}
\end{aligned}
$$

where $F \subset \subset G$ means that $F$ ranges over the finite subgraphs of $G$.

Observe that by this proposition, the conclusion of our Theorem 4 is non-trivial iff the Markov shift is SPR. 
6.2. Semi-local zeta function of large subsets. - The first step of the proof of Theorem 4 is the following weaker claim on semi-local zeta functions defined by large subgraphs:

Claim 6.2. - For every $\epsilon>0$, there exists a finite subset $F_{0} \subset \subset G$ such that for all finite subsets $F_{0} \subset F \subset \subset G$, the semi-local zeta function $\zeta_{F}^{G}(z)$ extends meromorphically to $|z|<\exp -\left(h_{\infty}(G)+\epsilon\right)$.

The crux of the proof is to check that

$$
\zeta_{F}^{G}(z)=1 / \operatorname{det}(\operatorname{Id}-L(z))
$$

with $L(z)$ a finite square matrix with holomorphic entries for $|z|<e^{-\left(h_{\infty}(G)+\epsilon\right)}$ and Id is the identity matrix.

One can give a direct, self-contained proof by generalizing an algebraic formula for the determinant of a finite matrix in terms of its block decomposition used for a similar purpose in [20. We give a shorter proof based on the formula in eq. (28), quoted from [2], as suggested by a referee.

Proof of Claim 6.2, Let $F$ be a finite subgraph of $G$. For each $u, v \in F$, define

$$
\begin{aligned}
& f_{n}^{F}(u, v):=\#\left\{x_{1} \ldots x_{n-1}: x \in \Sigma(G) \text { s.t. } x_{0}=u, x_{n}=v\right. \\
&\text { and } \left.\left\{x_{1}, x_{2}, \ldots, x_{n-1}\right\} \cap F=\emptyset\right\} .
\end{aligned}
$$

Recall the definition of $h(G \backslash F, G)$ as introduced in Proposition 4.10, There is $F_{0} \subset \subset$ $G$ such that, if $F_{0} \subset F \subset \subset G$, then $h(G \backslash F, G)<h_{\infty}(G)+\epsilon$ for arbitrarily small $\epsilon>0$. Hence, by eq. (26), for all $u, v \in F$ :

$$
\limsup _{n \rightarrow \infty} \frac{1}{n} \log f_{n}^{F}(u, v)<h_{\infty}(G)+\epsilon .
$$

Now define $L_{n}=L_{n}(z)$ to be the $F \times F$-matrix with following polynomial entries in $z$ :

Consider the zeta function:

$$
L_{n}(u, v):=\sum_{0 \leq k \leq n} f_{k}^{F}(u, v) z^{k} .
$$

$$
\begin{aligned}
\zeta_{n}^{F}(z):=\exp \sum_{p \geq 1} \frac{z^{p}}{p} \#\left\{x_{0} \ldots x_{p}: x \in \Sigma(G), \sigma^{p}(x)=x,\right. \\
\left.\left\{i \in \mathbb{Z}: x_{i} \in F\right\} \text { has gaps of lengths at most } n\right\} .
\end{aligned}
$$

The formula from $[2]$ is:

$$
\zeta_{n}^{F}(z)=\operatorname{det}\left(\mathrm{Id}-L_{n}(z)\right)^{-1} .
$$

Let $L$ be the $F \times F$ matrix with power series entries defined by $L(u, v):=\lim _{n \rightarrow \infty} L_{n}(u, v)$. The limit here is in the sense of formal power series. Increasing $n$ only adds high powers, hence this limit exists. Also $\zeta_{F}^{G}(z)=\lim _{n \rightarrow \infty} \zeta_{n}^{F}(z)$. By eq. (28), we get $\zeta_{F}^{G}(z)=\operatorname{det}(\operatorname{Id}-L(z))^{-1}$.

Eq. (27)implies that the entries of $L$ have a radius of convergence at least $e^{-h_{\infty}(G)-\epsilon}$. Therefore $\zeta_{F}^{G}(z)$ must be meromorphic over $|z|<\exp -\left(h_{\infty}(G)+\epsilon\right)$. 
6.3. Proof of Theorem 4, - Claim 6.2 shows the meromorphy of semi-local zeta functions relative to large finite subsets.

We first show the last claim of the Theorem. For $\epsilon>0$, let $F_{0} \subset \subset G$ as in the proof of Claim 6.2 let $F^{\prime}, F \supset F_{0}$ be other finite subgraphs. Let $F_{1}:=F \cup F^{\prime}$.

$$
\frac{\zeta_{F_{1}}^{G}(z)}{\zeta_{F}^{G}(z)}=\exp \sum_{n \geq 1} \frac{z^{n}}{n} \#\left\{x \in \Sigma: \sigma^{n}(x)=x \text { and }\left\{x_{0}, \ldots, x_{n-1}\right\} \text { meets } F_{1} \text { but not } F\right\} \text {. }
$$

The radius of convergence of the above series is at least $e^{-h\left(G \backslash F_{0}\right)} \geq e^{-\left(h_{\infty}(G)+\epsilon\right)}$. The same applies to $\zeta_{F_{1}}^{G}(z) / \zeta_{F^{\prime}}^{G}(z)$. This proves that $\zeta_{F}^{G}(z) / \zeta_{F^{\prime}}^{G}(z)$ is a holomorphic non-zero function over $|z|<e^{-\left(h_{\infty}(G)+\epsilon\right)}$.

We now show that all semi-local zeta functions are meromorphic on $|z|<e^{-h_{\infty}(G)}$, finishing the proof of Theorem 4 .

Let $F \subset \subset G$. Let $\epsilon>0$. By taking $H$ such that $F \subset H \subset \subset G, H$ large enough, we can ensure that

$$
h(G \backslash H, G \backslash F) \leq h_{\infty}(G \backslash F)+\epsilon
$$

(see the definition of $h(\cdot, \cdot)$ in Proposition 4.10). After possibly increasing $H$, Claim 6.2 ensures that $\zeta_{H \backslash F}^{G \backslash F}(z)$ has a meromorphic extension to $|z|<\exp -h_{\infty}(G \backslash F)-\epsilon$. We compute:

$$
\begin{aligned}
\zeta_{H}^{G}(z) / \zeta_{F}^{G}(z)= & \exp \sum_{n \geq 1} \frac{z^{n}}{n} \#\left\{x \in \Sigma(G): \sigma^{n} x=x\right. \text { and } \\
& \left.\left\{x_{0}, \ldots, x_{n-1}\right\} \cap H \neq \emptyset \text { but }\left\{x_{0}, \ldots, x_{n-1}\right\} \cap F=\emptyset\right\} \\
= & \exp \sum_{n \geq 1} \frac{z^{n}}{n} \#\left\{x \in \Sigma(G \backslash F): \sigma^{n} x=x\right. \text { and } \\
& \left.\left\{x_{0}, \ldots, x_{n-1}\right\} \cap(H \backslash F) \neq \emptyset\right\} \\
=\zeta_{H \backslash F}^{G \backslash F}(z) . &
\end{aligned}
$$

As $h_{\infty}(G \backslash H) \leq h_{\infty}(G \backslash F) \leq h_{\infty}(G)$ (see the remark after Definition 1.10). Thus $\zeta_{F}^{G}(z)=\zeta_{H}^{G}(z) / \zeta_{H \backslash F}^{G \backslash F}(z)$ is meromorphic on $|z|<\exp -h_{\infty}(G)-\epsilon$. Letting $\epsilon>0$ decrease to 0 , finish the proof of Theorem 4 .

\section{Proof of the Consequences}

7.1. Measures of maximum entropy. - Our Structure Theorem implies that the set of maximum measures for a $*$-QFT puzzle or for the associated Markov shift have the same cardinality. We apply some results of Gurevič.

First, according to 15, each irreducible subshift of a Markov shift carries at most one maximum measure and this measure, if it exists, is a Markov measure (which implies by [21] that it is a finite extension of a Bernoulli). Hence, a $*$-QFT puzzle has at most countably many maximum measures (because it has at most countably many states) and a QFT puzzle has only finitely many irreducible components (because 
its spectral decomposition contains finitely many irreducible subshifts with maximum entropy).

The existence of a maximum measure for a QFT puzzle follows from the fact that the spectral decomposition of its Markov diagram must contain an irreducible subshift with entropy equal to that of the puzzle and that this subshift must be SPR. SPR implies positive recurrence which is equivalent to the existence of a maximum measure by the same result of Gurevič. Theorem 2 is proved.

7.2. Zeta functions. - We prove Theorem 3 Recall that for the results involving the counting of the periodic points, we assume, in addition to QFT, determinacy. For simplicity, we assume that the Markov diagram $\mathcal{D}$ is irreducible and leave the general case to the reader. Let $\epsilon>0$. Theorem 1 gives a large integer $N$ such that the $n$-periodic orbits of $i_{N}\left(X_{V}\right)$ and the loops of $\mathcal{D}$ going through $\mathcal{D}_{N}$ can be identified up to an error bounded by $\exp \left(h_{\mathcal{C}}(V)+h_{\mathrm{wloc}}(V)+\epsilon\right) n$. Hence

$$
\zeta^{N}(z):=\exp \sum_{n \geq 1} \frac{z^{n}}{n} \#\left\{x \in i_{N}\left(X_{V}\right): F_{V}^{n}(x)=x\right\}
$$

is equal to the semi-local zeta function of $\mathcal{D}$ at $\mathcal{D}_{N}$ up to a holomorphic, non-zero factor on the disk $|z|<e^{-h_{\mathcal{C}}(V)-h_{\mathrm{wloc}}(V)-\epsilon}$ by Claim (3) of Theorem 1 By Theorem 4. this semi-local zeta function can be extended to a meromorphic function on $|z|<$ $e^{-h_{\mathcal{C}}(V)-h_{\text {wloc }}(V)}$, proving the main claim.

The singularities of $\zeta^{N}(z)$ on $|z|=e^{-h_{\mathrm{top}}(V)}$ are as claimed by the same statement proved for local zeta function ( $F$ reduced to one vertex) by Gurevic and Savchenko 17.

This concludes the proof of Theorem 3 ,

7.3. Equidistribution of periodic points. - We give a sketch of the proof of Theorem 5 which is essentially that from [11 using the estimates of the analysis of the zeta function above.

There is equidistribution for an irreducible SPR Markov shift according to Gurevic and Savchenko [17. For the (easy) extension to the general case, it is enough to see (like in [11]) that the number of $n$-periodic points living on an irreducible SPR Markov shift $\Sigma$ with period $p$ is equivalent to $p e^{n h_{\mathrm{top}}(\Sigma)}$ if $n$ is a multiple of $p$, zero otherwise.

To apply it to the puzzle, one has to recall the following facts from the above analysis of the zeta function:

- the projection $\Sigma(\mathcal{D}) \rightarrow \underline{X}_{V}$ is continuous;

- there is a one-to-one, period-preserving correspondence between $i_{N}$-projections of periodic points going through a large finite subset $F$ and a subset of the periodic points of $X_{V}$;

- the remaining periodic points both on $i_{N}\left(X_{V}\right)$ and $\Sigma(\mathcal{D})$ contributes negligibly to the considered measures by the reasoning in the proof of Theorem 3 , 


\section{Application to entropy-expanding maps}

We prove Theorem 7 , smooth entropy-expanding maps introduced in 7] define determined puzzles of quasi-finite type, provided that they are endowed with a good partition in the sense of section 1.7. We prove a more detailed statement (Theorem 8 and give some consequences in Corollaries 8.2,8.3. The first corollary is a new proof of results in 9 under an additional assumption. The second is new.

8.1. Puzzle and consequences. - At this point, $T$ may be just a continuous self-map of a compact metric space $M$ together with a finite partition $P$ into subsets $A$ such that $\bar{A}=\overline{\operatorname{int}(A)}$ and $T \mid \bar{A}$ is one-to-one. $\mathcal{P}$ is the set of the interiors of the elements of $P$.

The puzzle is defined by the refining sequence of "partitions" $\mathcal{P}_{n}$ which are, for each $n \geq 1$, the set of almost connected components of the $\mathcal{P}, n$-cylinders, i.e., intersections of the form $A_{0} \cap T^{-1} A_{1} \cap \cdots \cap T^{-n+1} A_{n-1}, A_{i} \in \mathcal{P}_{n}$. We assume that each $\mathcal{P}_{n}$ is finite. Their advantage over the usual connected components is the following key upper bound on the constraint entropy (to be proved later):

Proposition 8.1. - For the puzzle $V$ defined by almost connected components of the P-cylinders:

$$
h_{\mathcal{C}}(V) \leq h_{\text {top }}(T, \partial P)+h_{\text {mult }}(T, P)
$$

where $h_{\text {mult }}(T, P):=\lim \sup _{n \rightarrow \infty} \frac{1}{n} \log \operatorname{mult}\left(P^{n}\right)$ with $\operatorname{mult}(Q):=\max _{x \in M} \#\{A \in$ $Q: \bar{A} \ni x\}$.

We shall show that the puzzle defined in this way by an entropy-expanding map with a good partition is close to the original dynamics and also satisfies the remaining assumptions of our theory.

Remark. The above proposition is the counterpart of the upper bound on minimum left constraint entropy in [11], first claim in the proof of Lemma 7, p. 385. It is here that we reap the main benefit of the puzzle construction: we can consider almost connected components of cylinders, instead of whole cylinders - thus we get the direct link between the constraint entropy and the topological entropy of the boundary "for free", without having to assume the connectedness of cylinders as in [11, Lemma 7.

We recall some well-known notions to fix precise definitions and notations.

The coding map $\gamma_{V}$ of $\left(M, T,\left(\mathcal{P}_{n}\right)_{n \geq 1}\right)$ (or just the coding of $V$ ) is the partially defined map $\gamma: M^{\prime} \rightarrow X_{V}$ defined by (i) $M^{\prime}:=\bigcap_{n \geq 1} \bigcup_{A \in \mathcal{P}_{n}} A$; (ii) $\gamma(x)$ is the unique $y \in X_{V}$ such that, for all $n \geq 1, T^{n} x \in y_{n}$. The coding for the usual symbolic dynamics, simply denoted by $\gamma$, is obtained in this way by considering the partitions into cylinders of given order: $\mathcal{P}^{1}, \mathcal{P}^{2}, \ldots$

A finite extension of $F: X \rightarrow X$ is a skew product over $F$ with finite fibers, i.e., $G: Y \rightarrow Y$ such that $Y \subset X \times \mathbb{N}, \#(Y \cap\{x\} \times \mathbb{N})<\infty$ for all $x \in X$, and $G(x, n)=(F(x), \Psi(x, n))$ for some $\Psi: X \times \mathbb{N} \rightarrow \mathbb{N}$. We do not require the cardinality of the fibers to be constant.

A periodic extension of $F: X \rightarrow X$ is a map of the form $H: X \times\{0, \ldots, p-1\} \rightarrow$ $X \times\{0, \ldots, p-1\}$ with, for $0 \leq j<p, H(x, j)=H(x, j+1)$ and $H(x, p-1)=(F(x), 0)$. 
Theorem 8. - Let $T: M \rightarrow M$ be a $C^{\infty}$ entropy-expanding map of a compact manifold. Assume that $\mathcal{P}$ is a good partition and let $(V, i, f)$ be the puzzle obtained by taking the almost connected components of the $\mathcal{P}, n$-cylinders, $n \geq 0$ (see section 1.2). Let $\gamma_{V}$ be the coding,

Then:

1. $\gamma_{V}$ defines an entropy-conjugacy between $\left(X_{V}, F_{V}\right)$ and $(M, T)$, possibly up to a finite extension: there is a Borel finite extension $G$ of $F_{V}$ and an entropy conjugacy of $G$ and $T$ which extends $\gamma_{V}$;

2. $h_{\mathcal{C}}(V) \leq h^{d-1}(T)<h_{\text {top }}(T)=h_{\text {top }}(V)$;

3. $V$ is of quasi-finite type with $h_{\mathrm{wloc}}(V)=0$;

4. One can find a determined subpuzzle $V^{\prime} \subset V$ such that the two previous properties still hold and only few periodic orbits are destroyed:

$$
\forall N \geq 1 \limsup _{n \rightarrow \infty} \frac{1}{n} \log \#\left\{\xi \in i_{N}\left(X_{V}\right): \xi=F_{V}^{n}(\xi) \text { and } \xi \notin i_{N}\left(X_{V^{\prime}}\right)\right\} \leq h^{d-1}(T) .
$$

Applying Theorems 2 and 6 to $V$ yields a new proof of a slightly weaker version of our result $[\mathbf{9}$ about the measures of large entropy of entropy-expanding maps (we "lose" here a finite extension):

Corollary 8.2. - Let $T: M \rightarrow M$ be a $C^{\infty}$ entropy-expanding map. Let $\mathcal{P}$ be a good partition. Then:

- T has finitely many ergodic, invariant probability measure with maximum entropy;

- the natural extension of such maps T are classified up to entropy-conjugacy and possibly a period and a finite extension by their topological entropy.

Theorem 3 applied to $V^{\prime}$ gives information about periodic points:

Corollary 8.3. - In the same setting, let $\epsilon>0$. Perhaps after replacing $\mathcal{P}$ with a finer good partition, the Artin-Mazur zeta function at level $\mathcal{P}$ of $T$ :

$$
\zeta_{\mathcal{P}}(z):=\exp \sum_{n \geq 1} \frac{z^{n}}{n} \#\left\{\alpha \in \gamma\left(M^{\prime}\right): \sigma^{n} \alpha=\alpha\right\}
$$

is holomorphic on the disk $|z|<e^{-h_{\mathrm{top}}(T)}$ and has a meromorphic extension to the larger disk $|z|<e^{-h^{d-1}(T)-\epsilon}$. In particular, there exist integers $p \geq 1$ and $m \geq 1$ such that for $n \rightarrow \infty$ along the multiples of $p$ :

$$
\#\left\{\alpha \in \gamma\left(M^{\prime}\right): \sigma^{n} \alpha=\alpha\right\} \sim m e^{n h_{\mathrm{top}}(f)}
$$

Proof of Corollaries $8.2,8.3$, Corollary 8.2 is a trivial consequence of point 1 of Theorem 8 together with Theorems 2 and 6

Corollary 8.3 follows similarly from points 3 and 4 of Theorem 8 together with Theorem 3 using as the refined finite good partition, the partition defined by the almost connected components of the $\mathcal{P}, N$-cylinders where $N=N(\epsilon)$ is given by Theorem 3 , 
Remark. (1) If $M$ is one or two-dimensional, then a topological argument easily shows that each periodic sequence in the coding $\gamma\left(M^{\prime}\right)$ correspond to a periodic point (e.g., using Brouwer fixed point theorem in connected components of the closure of cylinders). In higher dimension, one must use the non-uniform expansion.

(2) The results of Kaloshin 22 show that upper bounds on the number of periodic points can hold for arbitrary maps only after some identifications.

In the sequel we prove Theorem 8 .

\subsection{Entropy-conjugacies. -}

Lemma 8.4. - Let $T: M \rightarrow M$ be an entropy-expanding map with a good partition $\mathcal{P}$. Then the puzzle defined by the almost connected components of cylinders has the same entropy as T. More precisely, the coding $\gamma_{V}$ defines an entropy-conjugacy between $T$ and a Borel finite extension of $F_{V}$.

To prove this, we use a common extension $X_{V} \ltimes M$ of the puzzle and of $T$ defined as:

$$
X_{V} \ltimes M=\overline{\left\{(v, x) \in X_{V} \times M: \forall n \geq 0 x \in v_{n}\right\}}
$$

endowed with the map $F_{V} \ltimes T$ which is just the restriction of the direct product. Let $\pi_{1}$, resp. $\pi_{2}$, be the projection $X_{V} \ltimes M \rightarrow X_{V}$, resp. $X_{V} \ltimes M \rightarrow M$.

- We claim that $F_{V} \ltimes T$ and $T$ are entropy-conjugate. Observe that, the partition being good for $T$, no point returns infinitely many times to $\partial P$. Hence $\partial P$ has zero measure w.r.t. any $T$-invariant probability measure. The same is true for $\pi_{2}^{-1}(\partial P)$. Hence $(v, x) \mapsto x$ is an isomorphism w.r.t. any invariant probability measure, proving the claim. In particular, $h_{\text {top }}\left(F_{V} \ltimes T\right)=h_{\text {top }}(T)$ by the variational principle.

- We claim that $F_{V} \ltimes T$ and $F_{V}$ are entropy-conjugate, perhaps after replacing the latter $F_{V}$ by a Borel finite extension. As the extension is continuous and compact, any invariant probability measure of $F_{V}$ can be lifted to $F_{V} \ltimes T$. We have to show that, given a large entropy measure of $F_{V}$ (1) there are only finitely many ergodic lifts $\hat{\mu}$; (2) for each such $\hat{\mu}, \pi_{1}:\left(X_{V} \ltimes M, \hat{\mu}\right) \rightarrow\left(X_{V}, \pi_{1} \hat{\mu}\right)$ is a finite extension.

We first prove point (2). We can assume $\hat{\mu}$ to be an arbitrary $F_{V} \ltimes T$-invariant and ergodic probability measure with $h\left(F_{V} \ltimes T, \hat{\mu}\right)=h\left(T, \pi_{2} \hat{\mu}\right)>h^{d-1}(T)$. Let $\mu=\left(\pi_{2}\right)_{*} \hat{\mu}$ and $\nu=\left(\pi_{1}\right)_{*} \hat{\mu}$. $\mu$ is a $T$-invariant ergodic measure satisfying $h(T, \mu)>h^{d-1}(T)$. By 9 this implies that $\mu$ has only strictly positive Lyapunov exponents, hence, by [10], $\pi_{1}:\left(X_{V} \ltimes M, \hat{\mu}\right) \rightarrow\left(X_{V}, \nu\right)$ is a finite extension. This proves point (2).

We prove point (1) following [10. Assume by contradiction that there exists infinitely many distinct ergodic lifts $\hat{\mu}_{1}, \hat{\mu}_{2}, \ldots$ of some ergodic and invariant probability measure $\mu$ of $F_{V}$. We can assume that $\hat{\mu}_{n}$ converges to some $\hat{\mu}_{*}$. As $\pi_{2}$ is continuous, $\hat{\mu}_{*}$ is also a lift of $\mu$ and so are almost all of its ergodic components. They project on $M$ to ergodic invariant probability measures with positive Lyapunov exponents. As explained in 10, this implies that for each such ergodic component $\hat{\nu}$, for $\hat{\nu}$-a.e. $(v, x)$, there exists a ball $B$ around $x$ in the fiber which contains no generic point wrt any measure distinct from $\hat{\nu}$. It follows that there are only countably (or finitely) many ergodic components. Thus, there exists an ergodic component of $\hat{\mu}_{*}$, such that 
the union of these fibered neighborhood has positive $\hat{\mu}_{*}$-measure. Hence it has positive measure for $\hat{\mu}_{n}$ for $n$ large. But this implies that $\hat{\mu}_{n}=\hat{\mu}_{*}$, a contradiction. Point (1) is proven and the claim follows.

The above two claims prove the lemma.

8.3. Constraint entropy. - Before proving Proposition 8.1 which will imply claim 2 of Theorem 8 , we give a geometric necessary condition for the irreducibility of puzzle pieces.

Lemma 8.5. - Let $(V, i, f)$ be a puzzle generated by the almost connected components of the cylinders of a partition $\mathcal{P}$.

Let $v \in V$ and let $A$ be the unique the element of $\mathcal{P}$ containing $v$,

$$
\overline{f(v)} \cap \partial T(A)=\emptyset \Longrightarrow v \text { is } f \text {-reducible. }
$$

Proof: Assume $\overline{f(v)} \cap \partial T(A)=\emptyset . \quad v$ is an almost connected component of $A \cap$ $T^{-1}(f(v))=(T \mid \bar{A})^{-1}(f(v))$. By the assumption, this last set is uniformly homeomorphic to $f(v)$, hence is almost connected. Therefore it is equal to $v$.

This shows that $v$ is uniquely determined by $f(v)$ and $A=i_{1}(v)$ (a fortiori $i(v)$ ), verifying condition (2) of reducibility.

Consider now $\left(^{*}\right) f: \mathcal{T}_{i}(v) \rightarrow \mathcal{T}_{i}(f(v))$. Observe that for any $w \in \mathcal{T}_{i}(f(v))$, $w \subset f(v)$. Hence, $\bar{w} \cap \partial T(A)=\emptyset$. The reasoning for the uniqueness of $v$ shows that the map $\left(^{*}\right)$ is one-to-one: $f(u)=f\left(u^{\prime}\right)$ implies that $T(u)$ and $T\left(u^{\prime}\right)$ are both almost connected subsets of $f(u)=f\left(u^{\prime}\right)$, so they must be equal.

For $w \in \mathcal{T}_{i}(f(v)), u=(T \mid A)^{-1}(w) \in \mathcal{T}_{i}(v)$ satisfies $w=f(u)$. Hence the map (*) is onto and therefore an isomorphism, proving condition (1) of reducibility.

Proof of Proposition 8.1; Let $r>0$ and $\epsilon>0$. Let $\Sigma_{n}$ be an arbitrary $(r, n)$ separated subset of $\mathcal{C}_{n}$, the set of irreducible pieces of order $n$. Recall that there exists some $L=L(r)$, such that, for all $n \geq L, x, x^{\prime} \in V_{n}$ are $(r, n)$-separated then there exists some $0 \leq k<n-L$ such that $\left(f^{k} x\right)_{L} \neq\left(f^{k} x^{\prime}\right)_{L}$.

We are going to bound the cardinality of $\Sigma_{n}$ by $e^{\left(h_{\mathrm{top}}(T, \partial \mathcal{P})+h_{\mathrm{mult}}(T, P)+2 \epsilon\right) n}$.

Let $\rho>0$ be smaller than the distance between any two almost connected component of any $L$-cylinder (there are only finitely many of them, $L$ being fixed, and the distance between any two of them is positive as we are considering almost connected components). For all integers $n$ large enough, $\operatorname{mult}\left(\mathcal{P}^{n}\right)<e^{\left(h_{\text {mult }}(T, P)+\epsilon\right) n}$ and $r(\rho / 2, n, \partial \mathcal{P}) \leq e^{\left(h_{\text {top }}(T, \partial \mathcal{P})+\epsilon\right) n}$.

Let $S_{n}$ be a minimum $(\rho / 2, n)$-spanning subset of $\partial P$. To every $v \in \Sigma_{n}$, associate a point $x=x(v) \in S_{n}$ such that $d\left(T^{k} v, T^{k} x\right)<\rho / 2$ for all $0 \leq k<n\left(T^{k} v\right.$ is a subset of $M)$. This is possible since $\bar{v} \cap \partial P \neq \emptyset$ by Lemma 8.5 .

The map $x: \Sigma_{n} \rightarrow S_{n}$ is at most $\left(\# V_{L}\right)^{L} \cdot e^{\left(h_{\text {mult }}(T, P)+\epsilon\right) n}$-to-1. Indeed, assume that there exists $x \in S_{n}$ with more than this number of pre-images. We can find a set of $e^{\left(h_{\text {mult }}(T, P)+\epsilon\right) n}$ pre-images, all with the same $\left(\left(f^{k} v^{\prime}\right)_{L}\right)_{n-L \leq k<n}$. As $e^{\left(h_{\text {mult }}(T, P)+\epsilon\right) n}>$ $\operatorname{mult}\left(\mathcal{P}^{n}\right)$, two of these, say $v$ and $v^{\prime}$, must almost connected components of the same $n, \mathcal{P}$-cylinder. But then $d\left(T^{k} v, T^{k} v^{\prime}\right) \leq d\left(T^{k} v, T^{k} x\right)+d\left(T^{k} x, T^{k} v^{\prime}\right)<\rho$ implies 
$\left(f^{k} v^{\prime}\right)_{L}=\left(f^{k} v\right)_{L}$ for all $0 \leq k<n-L$, contradicting the separation assumption. Therefore $\# \Sigma_{n} \leq\left(\# V_{L}\right)^{L} \cdot e^{\left(h_{\text {mult }}(T, P)+\epsilon\right) n} \# S_{n}$ and

$$
h_{\mathcal{C}}\left(F_{V}\right) \leq h_{\text {top }}(T, \partial \mathcal{P})+h_{\text {mult }}(T, P)+2 \epsilon,
$$

with arbitrary $\epsilon>0$, proving the claim.

8.4. Determinacy. - We turn to determinacy. The delicate point here is that it is possible (though exceptional) that $u \succeq_{f} v$ in the absence of the geometric property of Lemma 8.5. because of the following phenomenon.

A puzzle piece $v \in V$ is trivial if there exists $k \geq 1$ such that for every $w \in \mathcal{T}_{i}(v)$,

$$
w \cap T\left(\partial \mathcal{P}^{k}\right) \neq \emptyset \text {. }
$$

The trivial subset of $V$ is the smallest subset $V^{0}$ of the puzzle such that:

$-V^{0}$ contains all trivial pieces;

- if $f(v) \in V^{0}$ then $v \in V^{0}$.

Observe that $V \backslash V^{0}$ equipped with the restrictions of $i$ and $f$ is a puzzle as $f\left(V \backslash V^{0}\right) \subset$ $V \backslash V^{0}$ (by definition) and $i\left(V \backslash V^{0}\right) \subset V \backslash V^{0}$ (as $\left.\mathcal{T}_{i}(i(v)) \supset \mathcal{T}_{i}(v)\right)$.

Lemma 8.6. - Let $(V, i, f)$ be the puzzle defined by a dynamical system $T: M \rightarrow M$ as in Proposition 8.1. The non trivial puzzle $V^{\prime}:=V \backslash V^{0}$ is determined.

Assume additionally that $h_{\mathcal{C}}(V)<h_{\mathrm{top}}(V)$. Then the obvious injection $i: X_{V^{\prime}} \rightarrow$ $X_{V}$ is an entropy-conjugacy and the approximate periodic points of the two systems satisfy the estimate (30) of Theorem [8:

$$
\forall N \geq 1 \limsup _{n \rightarrow \infty} \frac{1}{n} \log \#\left\{\xi \in i_{N}\left(X_{V}\right) \backslash i_{N}\left(X_{V^{\prime}}\right): \sigma^{n} \xi=\xi\right\} \leq h_{\mathcal{C}}(V) .
$$

Proof: To prove the determinacy, we consider $v, v^{\prime}, w \in V^{\prime}$ such that $i_{1}(v)=$ $i_{1}\left(v^{\prime}\right)=: A \in \mathcal{P}$ and $v, v^{\prime} \succeq_{f}^{1} w$. Assume by contradiction that $v \neq v^{\prime} . T \mid \bar{A}$ is a homeomorphism hence $v, v^{\prime}$ are disjoint almost connected components of $(T \mid \bar{A})^{-1}(T A \cap w)$. $v, v^{\prime} \succeq_{f} w$ implies that:

$$
\left\{f(u): u \in \mathcal{T}_{i}(v)\right\}=\left\{f\left(u^{\prime}\right): u^{\prime} \in \mathcal{T}_{i}\left(v^{\prime}\right)\right\}=\mathcal{T}_{i}(w) .
$$

Hence, every $t \in \mathcal{T}_{i}(w)$ is an almost connected set containing both disjoint sets $T(v)$ and $T\left(v^{\prime}\right)$. Therefore $t \cap \partial T(v) \neq \emptyset$, so that $t \cap T\left(\partial \mathcal{P}^{|v|}\right) \neq \emptyset$. Thus $w$ is trivial, the sought-for contradiction.

We now let $\mu$ be an ergodic $F_{V}$-invariant probability measure such that, for some $v \in V^{0}, \mu\left([v]_{V}\right)>0$. By invariance of $\mu, \mu\left([w]_{V}\right)>0$ for a trivial $w=f^{n}(v)$ with $0 \leq n<|v|$. Now, $x \in[w]_{V}$ implies that $x_{|w|}=w$ and $x_{n} \cap T\left(\partial \mathcal{P}^{\ell}\right) \neq \emptyset$ for all $n \geq|w|$ and some fixed, minimal $\ell \geq 0$. Therefore $t:=F_{V}^{\ell}(x)$ satisfies $t_{m} \cap T(\partial P)$ for all $m \geq|w|-\ell$. The reasoning in the proof of Proposition 8.1 implies:

$$
h\left(F_{V}, \mu\right) \leq h_{\mathrm{top}}\left(F_{V},[w]_{V}\right) \leq h_{\mathcal{C}}(V)<h_{\mathrm{top}}(V),
$$

proving the entropy-conjugacy.

Consider now some periodic sequence $\xi \in i_{N}\left(X_{V}\right) \backslash i_{N}\left(X_{V^{\prime}}\right)$. Hence $\xi=i_{N}(x)$ with $x_{m} \in V^{0}$ for some $m \geq 0$. Thus $f^{n}\left(x_{m}\right)$ is trivial. We may assume $n=0$ by 
shifting to another point of the same periodic orbit). Therefore $x_{p} \cap T\left(\partial \mathcal{P}^{k}\right) \neq \emptyset$ for some $k$ and all $p \geq m$. As above, it follows that $f^{j}\left(x_{p+j}\right) \cap T \partial \mathcal{P} \neq \emptyset$ for all $p \geq m$ and some $j$ which can be assumed to be fixed and then 0 . The claimed bound on the number of periodic points follows.

8.5. W-local Entropy. — We prove the third point of Theorem 8

Lemma 8.7. - If $V$ is a *-QFT puzzle which is determined then, for all invariant and ergodic probability measures $\mu$ on $X_{V}$ with $h\left(F_{V}, \mu\right)>h_{\mathcal{C}}(V)$,

$$
h\left(F_{V}, \mu\right)=h\left(F_{V}, \mu, \epsilon_{*}\right)
$$

So in particular, $h_{\mathrm{wloc}}(V)=0$.

Proof: Any ergodic invariant probability measure on $X_{V}$ with entropy $>h_{\mathcal{C}}(V)$ can be lifted to an isomorphic $\hat{\mu}$ on $\Sigma(\mathcal{D})$ by Theorem $\left.1 . \sigma^{-1}, \hat{\mu}\right)=h(\sigma, \hat{\mu})=h\left(F_{V}, \mu\right)$ can be bounded by the growth rate of the number paths on $\mathcal{D}$ ending at any fixed vertex $v_{*} \in \mathcal{D}$ with $\hat{\mu}\left([v]_{\Sigma(\mathcal{D})}\right)>0$. But those paths are uniquely determined by their $i_{1}$-projection as $V$ is determined (Lemma 5.1). Thus, $h\left(F_{V}, \mu\right)=h\left(\sigma, i_{1}(\mu)\right)=$ $h\left(F_{V}, \mu, 1 / 2\right)$.

Corollary 8.8. - Let $V$ be a $*$-QFT puzzle with a subpuzzle $V^{\prime}$ which is determined. Assume that the inclusion $X_{V^{\prime}} \rightarrow X_{v}$ is a conjugacy with respect to all ergodic invariant probability measures with entropy $>h_{\mathcal{C}}(V)$. Then $h_{\mathrm{wloc}}(V)=0$.

Proof: By the previous lemma, $h_{\text {wloc }}\left(V^{\prime}\right)=0$. Let us see that this property carries over to $V$.

Let $\mu$ be an ergodic invariant probability measure of $X_{V}$ with $h\left(F_{V}, \mu\right)>h_{\mathcal{C}}(V)$. Hence, it can be identified to an invariant measure $\mu^{\prime}$ of $F_{V^{\prime}}$. Therefore $h\left(F_{V^{\prime}}, \mu^{\prime}\right)=$ $h\left(\sigma, i_{N}\left(\mu^{\prime}\right)\right)$ for some integer $N \geq 1$. But $V^{\prime} \subset V$ hence one can define almost everywhere $i_{N}: X_{V} \rightarrow i_{N}\left(X_{V^{\prime}}\right)$ and check that $i_{N}(\mu)$ and $i_{N}\left(\mu^{\prime}\right)$ are isomorphic so that $h\left(F_{V}, \mu\right)=h\left(\sigma, i_{N}(\mu)\right)$, proving the claim.

\section{A}

\section{Varying Radius of Meromorphy}

Definition A.1. - Denote by $\mathbf{M}(f)$ the radius of meromorphy of a formal power series $f$. It is zero if the radius of convergence of $f, \rho(f)$, is zero. Otherwise it is the supremum of the radiuses $r$ of the disks $D(r)$ centered at zero for which there exists a rational function $F(z)$ such that $f(z) / F(z)$ can be extended to a holomorphic and non-zero function on $D(r)$.

Fact A.2. - There exists a countable oriented $S P R$ graph $G \ni a, b$ such that $\mathbf{M}\left(\zeta_{a}^{G}\right) \neq$ $\mathbf{M}\left(\zeta_{b}^{G}\right)$.

We found this example after an illuminating discussion with O. Sarig. 
Before giving our construction, we recall some basic tools. The main tool here is the notion of a loop graph (or petal graph in the terminology of B. Gurevič). These graphs have a distinguished vertex and an arbitrary number of first return loop:(5) of each length based at the distinguished vertex, but distinct first return loops are disjoint except for the distinguished vertex. Such graphs are completely described by their first return series $f(z):=\sum_{n>1} f_{n} z^{n}$ where $f_{n}$ is the number of first return loops of length $n$ (based at the distinguished vertex). It is well-known that the local zeta function at the distinguished vertex is

$$
\zeta_{*}^{G}(z)=\frac{1}{1-f(z)}=\sum_{n \geq 1} \ell_{n} z^{n}
$$

where $\ell_{n}$ is the number of loops of length $n$ based at the distinguished vertex

We now give the construction. We consider two disjoint loop graphs defined by first return series $a(z):=\sum_{n \geq 1} a_{n} z^{n}$ and $b(z):=\sum_{n \geq 1} b_{n} z^{n}$. We call their respective distinguished vertices $a$ and $\bar{b}$.

We define a new graph $G$ by taking the disjoint union of:

- the two preceding loop graphs;

- a set of disjoint paths from $a$ to $b$ described by a series $s(z):=\sum_{n \geq 1} s_{n} z^{n}$ (there are $s_{n}$ simple paths of length $n$ from $a$ to $b$ and these are disjoint);

- a set of simple paths (i.e., injective as maps) from $b$ to $a$ described by a series $t(z):=\sum_{n \geq 1} t_{n} z^{n}$.

Claim A.3. - The first return series of $G$ at a is:

$$
\hat{a}(z)=a(z)+\frac{s(z) t(z)}{1-b(z)} .
$$

Indeed, any first return loop at $a$ in $G$ is exactly in one of the following classes:

- the first return loops in the loop graph $a$;

- the concatenations of a transition from $a$ to $b$, a (not necessarily first return) loop at $b$, a transition from $b$ to $a$.

Fix $b(z)=2 z^{2}$ (so the associated Markov shift is the set of all infinite concatenations of the two words of length 2 , say $b 0$ and $b 1$ ).

Let $\tau(z):=\sum_{n \geq 1} \tau_{n} z^{n}=s(z) t(z)$. We arrange it so:

$-\tau_{0}=\tau_{1}=0, \tau_{n}=0$ or 1 ;

- $|z|=1$ is the natural boundary of $\tau$.

By the Pólya-Carlson theorem [32, the last condition is equivalent to $\tau_{n}$ not being eventually periodic. This can be obtained by taking $\left\{n: s_{n}=1\right\}$ and $\left\{n: t_{n}=1\right\}$ to be disjoint subsets of $2,2^{2}, 2^{3}, \ldots$ satisfying the aperiodic condition above. It follows that $q(z):=\tau(z) /(1-b(z))$ satisfies:

1. $q_{0}=0,0 \leq q_{n} \leq 2^{n / 2+1} \leq 5^{n}$

${ }^{(5)}$ That is, sequences $v_{0} \rightarrow^{e_{1}} v_{1} \rightarrow \cdots \rightarrow^{e_{n}} v_{n}$ where the vertices $v_{i}$ 's and edges $e_{i}$ 's are distinct except for $v_{0}=v_{n}$ which is the distinguished vertex.

${ }^{(6)}$ These loops may go several times through the distinguished vertex. 
2. $|z|=1$ is the natural boundary of $q$.

Now, set $a_{0}=0$ and, for $n \geq 1: a_{n}:=5^{n}-q_{n} \geq 0$. We have: $\hat{a}_{n}=a_{n}+q_{n}=5^{n}$ for $n \geq 1, \hat{a}_{0}=0$. Hence $\hat{a}(z)=5 z /(1-5 z)$ and

$$
\zeta_{a}^{G}(z)=\frac{1}{1-\hat{a}(z)}=\frac{1-5 z}{1-10 z}
$$

is a rational function. In particular, $\mathbf{M}\left(\zeta_{a}^{G}\right)=\infty$. On the other hand,

$$
\hat{b}(z)=b(z)+\frac{\tau(z)}{1-a(z)}=2 z^{2}+\frac{\left(1-2 z^{2}\right) q(z)}{1-\frac{5 z}{1-5 z}+q(z)}=2 z^{2}+\frac{\left(1-2 z^{2}\right)(1-5 z)}{1+(1-10 z) / q(z)}
$$

Therefore $\zeta_{b}^{G}(z)=1 /(1-\hat{b}(z))$ has meromorphy radius: $\mathbf{M}\left(\zeta_{b}^{G}\right)=\mathbf{M}(\hat{b})=\mathbf{M}(q)=1$ and

$$
\mathbf{M}\left(\zeta_{b}^{G}\right)<\mathbf{M}\left(\zeta_{a}^{G}\right)
$$

as claimed.

Observe that $h(G)=\log 10$ and $h_{\infty}(G)=\log 5$. Hence $h_{\infty}(G)<h(G)$ and $G$ is SPR as claimed, finishing the construction.

\section{B}

\section{Good Partitions for Almost All Couplings}

We consider the following, convenient family of coupled maps. For $(a, b, c) \in \mathbb{R}^{3}$, we let

$$
F_{a, b, c}(x, y)=\left(a\left(1-4 x^{2}\right)+c y^{2}-\frac{1}{2}, b\left(1-4 y^{2}\right)+c x^{2}-\frac{1}{2}\right) .
$$

For $(a, b, c) \in \Omega=\left\{(a, b, c) \in(0,1)^{3}: c<4-4 \max (a, b)\right\}, F_{a, b, c}(Q) \subset Q$ for $Q:=\left[-\frac{1}{2}, \frac{1}{2}\right]^{2}$. There is a natural partition $\mathcal{P}$ into four elements:

$$
Q_{\epsilon_{1}, \epsilon_{2}}:=\left\{(x, y) \in Q: \epsilon_{1} x>0, \epsilon_{2} y>0\right\} \quad\left(\epsilon_{1}, \epsilon_{2}\right) \in\{-1,1\}^{2}
$$

according to the signs of $x$ and $y$. Most of the properties of a good partition are obvious for this convenient family:

Indeed, $F_{a, b, c} \mid \bar{Q}_{\epsilon_{1}, \epsilon_{2}}$ is obviously one-to-one. The boundary of the partition is:

$$
\partial \mathcal{P}=[-1 / 2,1 / 2] \times\{0\} \cup\{0\} \times[-1 / 2,1 / 2] \cup \partial Q .
$$

$\partial \mathcal{P}$ is obviously the image of a compact subset of $\mathbb{R}$ by a $C^{\infty}$ map. The semi-algebraic nature of both $F_{a, b, c}$ and $\partial \mathcal{P}$ implies that each cylinder has indeed finitely many connected components and therefore finitely many almost connected components.

To conclude, we show that, after discarding countably many hypersurfaces in the parameter space, there is a constant such that:

$$
\forall(x, y) \in Q \#\left\{k \geq 0: F_{a, b, c}^{k}(x, y) \in \partial \mathcal{P}\right\} \leq 2 .
$$

We prove that for each $0<n<m$, there exists a hypersurface containing all the parameters $(a, b, c)$ such $F_{a, b, c}^{n}(x, 0)$ and $F_{a, b, c}^{m}(x, 0)$ are both in $\{0\} \times[-1 / 2,1 / 2]$ for some $x \in[-1 / 2,1 / 2]$. The cases involving other pieces of $\partial \mathcal{P}$ are similar and together they imply eq. (32). 
Observe that $F_{a, b, c}^{k}(x, 0) \in\{0\} \times[-1 / 2,1 / 2]$ is equivalent to

$$
P_{k, a, b, c}(x)=0
$$

for some polynomials in $x$ whose coefficients are themselves polynomials in $a, b, c$.

The degrees of $P_{n, a, b, c}$ and $P_{m, a, b, c}$ are fixed, say $p$ and $q$, outside of an algebraic hypersurface. Hence the parameters we are considering are such that the $(p+q) \times$ $(p+q)$ resultant of the two polynomials $P_{n, a, b, c}$ and $P_{m, a, b, c}$ is zero: these parameters satisfy a polynomial equation. This equation is not trivial as it is not satisfied for $a=b=1, c=0$. Indeed, $F_{1,1,0}(x, y)=\left(\frac{1}{2}-4 x^{2}, \frac{1}{2}-4 y^{2}\right)$ so the $x$-coordinate can take the value 0 only once in an orbit (the subsequent values are then $1 / 2,-1 / 2,-1 / 2, \ldots$ ).

\section{References}

[1] V. Berthé, Sequences of low complexity: automatic and Sturmian sequences, Topics in symbolic dynamics and applications (Temuco, 1997), 1-34, London Math. Soc. Lecture Note Ser., 279, Cambridge Univ. Press, Cambridge, 2000.

[2] L. Block, J. Guckenheimer, M. Misiurewicz, L.-S. Young, Periodic points and topological entropy of one dimensional maps, in Global Theory of Dynamical Systems, (Lecture Notes in Math. 819), Springer, Berlin 1980, pp. 18-34

[3] M. Boyle, J. Buzzi, R. Gomez, Almost isomorphism of countable state Markov shifts, Journal fur die reine und angewandte Mathematik 592 (2006), 23-47.

[4] R. Bowen, Topological entropy for noncompact sets, Trans. A.M.S. 184 (1975), 125136.

[5] B. Branner, J.H. Hubbard, The iteration of cubic polynomials, Part II: Patterns and parapatterns, Acta Math. 169 (1992), 229-325.

[6] J. Buzzi, Intrinsic ergodicity of smooth interval maps, Israel J. Math. 100 (1997), $125-161$.

[7] J. Buzzi, Ergodicité intrinsèque de produits fibrés d'applications chaotiques unidimensionelles, Bull. Soc. Math. France 126 (1998), no. 1, 51-77.

[8] J. Buzzi, Markov extensions for multi-dimensional dynamical systems, Israel J. Math. 112 (1999), 357-380.

[9] J. Buzzi, On entropy-expanding maps, preprint 2000.

[10] J. Buzzi, The coding of non-uniformly expanding maps with an application to endomorphisms of $C P^{k}$, Ergodic Th. and Dynam. Syst. 23 (2003), 1015 - 1024.

[11] J. Buzzi, Subshifts of quasi-finite type, Invent. Math. 159 (2005), no. 2, 369-406.

[12] J. Buzzi, S. Ruette, Large entropy implies existence of a maximal entropy measure for interval maps, Discrete Contin. Dyn. Syst. 14 (2006), no. 4, 673-688.

[13] D. Fiebig, U.-R. Fiebig, M. Yuri, Pressure and equilibrium states for countable state Markov shifts, Israel J. Math. 131 (2002), 221-257.

[14] B.M. Gurevič, Topological entropy of a countable Markov chain, Dokl. Akad. Nauk SSSR 187 (1969), 715-718 - English: Soviet Math. Dokl. 10 (1969), 911-915.

[15] B.M. Gurevič, Shift entropy and Markov measures in the space of paths of a countable graph, Dokl. Akad. Nauk SSSR 192 (1970), 963-965 - English: Soviet Math. Dokl. 11 (1970), 744-747.

[16] B.M. Gurevič, Stably recurrent nonnegative matrices, Uspekhi Mat. Nauk 51 (1996), no. 3(309), 195-196 - English: Russian Math. Surveys 51 (1996), no. 3, 551-552 
[17] B.M. Gurevič, S. Savchenko, Thermodynamic formalism for symbolic Markov chains with a countable number of states, Uspekhi Mat. Nauk 53 (1998), no. 2(320), 3-106; - English: Russian Math. Surveys 53 (1998), no. 2, 245-344.

[18] B.M. Gurevič, Zargaryan, Gurevich, B. M.; Zargaryan, A. S. Conditions for the existence of a maximal measure for a countable symbolic Markov chain, Vestnik Moskov. Univ. Ser. I Mat. Mekh. (1988), , no. 5, 14-18, 103; English: Moscow Univ. Math. Bull. 43 (1988), no. 5, 18-2.

[19] F. Hofbauer, On intrinsic ergodicity of piecewise monotonic transformations with positive entropy, Israel J. Math. 34 (1979), no. 3, 213-237 (1980).

[20] F. Hofbauer, G. Keller, Zeta-functions and transfer-operators for piecewise linear transformations, J. Reine Angew. Math. 352 (1984), 100-113.

[21] Sh. Ito, H. Murata, H. Totoki, Remarks on the isomorphism theorem for weak Bernoulli transformations in the general case, Publ. Res. Inst. Math. Sci. 7 (1971/1972), 541-580.

[22] V. Kaloshin, Generic diffeomorphisms with superexponential growth of number of periodic orbits, Comm. Math. Phys. 211 (2000), no. 1, 253-271.

[23] A. Katok, Lyapunov exponents, entropy and periodic orbits for diffeomorphisms, Inst. Hautes Etudes Sci. Publ. Math. No. 51 (1980), 137-173.

[24] B. P. Kitchens, Symbolic dynamics. One-sided, two-sided and countable state Markov shifts, Universitext. Springer-Verlag, Berlin, 1998.

[25] D. Lind, B. Marcus, An introduction to symbolic dynamics and coding, Cambridge University Press, Cambridge, 1995.

[26] R. D. Mauldin, M. Urbański, Graph directed Markov systems. Geometry and dynamics of limit sets, Cambridge Tracts in Mathematics, 148. Cambridge University Press, Cambridge, 2003.

[27] C. T. McMullen, Complex dynamics and renormalization, Annals of Mathematics Studies, 135. Princeton University Press, Princeton, NJ, 1994.

[28] W. de Melo, S. van Strien, One-dimensional dynamics, Ergebnisse der Mathematik und ihrer Grenzgebiete (3), 25. Springer-Verlag, Berlin, 1993.

[29] J. Milnor, W. Thurston, On iterated maps of the interval, in: Dynamical Systems, Lecture Notes in Mathematics 1342, Springer, 465-564, 1988.

[30] M. Misiurewicz, Topological conditional entropy, Studia Math. 55 (1976), no. 2, 175-200.

[31] M. J. Pacifico, J. Vieitez, Entropy-expansiveness and domination, preprint IMPA no. D029 (2006). See http://www.preprint.impa.br

[32] R. Remmert, L. Kay, Classical Topics in Complex Function Theory, Graduate Texts in Mathematics, Springer, 1998.

[33] S. Ruette, Mixing $C^{r}$ maps of the interval without maximal measure, Israel J. Math. 127 (2002), 253-277.

[34] S. Ruette, On the Vere-Jones classification and existence of maximal measures for countable topological Markov chains, Pacific J. Math. 209 (2003), no. 2, 366-380.

[35] O. Sarig, Thermodynamic formalism for countable Markov shifts, Ergodic Theory Dynam. Systems 19 (1999), no. 6, 1565-1593.

[36] O. Sarig, Thermodynamic formalism for null recurrent potentials, Israel J. Math. 121 (2001), 285-311.

[37] O. Sarig, Phase Transitions for Countable Topological Markov Shifts, Commun. Math. Phys. 217 (2001), 555-577. 
[38] M. Viana, Multidimensional nonhyperbolic attractors, Inst. Hautes Etudes Sci. Publ. Math. No. 85 (1997), 63-96.

[39] P. Walters, An introduction to ergodic theory. Graduate Texts in Mathematics, 79. Springer-Verlag, New York-Berlin, 1982.

JÉrôme Buzzi, C.N.R.S. / Université Paris-Sud, 90405 Orsay cedex, France E-mail : jerome.buzzi@math.u-psud.fr • Url : www.jeromebuzzi.com 